\title{
THE LARGE, BRIGHT QSO SURVEY. V. QSOS IN THREE SOUTHERN FIELDS ${ }^{1}$
}

\author{
SimON L. MORRIS AND RAY J. WEYMANN \\ The Observatories of the Carnegie Institution of Washington, 813 Santa Barbara Street, Pasadena, California 91101
}

SCOTT F. ANDERSON

Department of Astronomy, FM-20, University of Washington, Seattle, Washington 98195

Paul C. Hewett

Institute of Astronomy, Madingley Road, Cambridge CB3 OHA, England

Craig B. Foltz and Frederic H. ChaffeE ${ }^{2}$

Multiple Mirror Telescope Observatory, University of Arizona, Tucson, Arizona 85721

Paul J. Francis ${ }^{3}$

Institute of Astronomy, Madingley Road, Cambridge CB3 OHA, England

Gordon M. MACAlPine

Department of Astronomy, University of Michigan Ann Arbor, Michigan 48109

Received 3 June 1991; revised 8 July 1991

\begin{abstract}
This is the fifth and final paper in a series reporting the results of a program aimed at selecting $\sim 1000$ QSOs brighter than $B_{J} \simeq 18.75$ using machine-scanned direct and objective-prism plates from the UK Schmidt Telescope. The plate material is scanned at the Automated Plate Measuring facility. The candidate list is derived using a number of complementary selection algorithms ranging from "traditional" criteria, such as the presence of strong emission features, to criteria designed to select objects whose objective-prism spectra cannot be classified as normal stars, although they may possess no obvious QSO-like features. Follow-up spectroscopy at the $2.5 \mathrm{~m}$ duPont telescope of the Las Campanas Observatory is used to classify each candidate. In this paper we present results from three UK Schmidt Telescope fields in which 200 QSOs have been found, 182 of which form part of the LBQS sample. Coordinates, magnitudes, redshifts, and spectra of moderate resolution and signal-to-noise ratio are given for all 200 QSOs as well as for 3 additional extragalactic objects which fail to meet our absolute magnitude criterion as QSOs.
\end{abstract}

\section{INTRODUCTION}

This is the last paper in a series of five presenting the observational data for the Large, Bright QSO Survey (LBQS), which is aimed at providing a sample of more than 1000 QSOs of a wide variety of types in the range $16.0 \leqslant B_{J} \leqslant 18.85$. Candidates are selected from machinescanned UK Schmidt Telescope direct and objective-prism plates using well-defined and consistently applied algorithms, and follow-up spectroscopy is obtained on the MMT and the $2.5 \mathrm{~m}$ duPont telescope. The reader is referred to the previous four papers in the series (Foltz et al. 1987; Foltz et al. 1989; Hewett et al. 1991; Chaffee et al. 1991, Papers I-IV, respectively) for a description of the scientific objectives, and candidate selection criteria used for the LBQS.

This paper reports observations of QSO candidates in the final three fields of the LBQS, all of which are located south of the celestial equator. Section 2 describes the observations and presents coordinates, magnitudes, and spectra for each

${ }^{1}$ Observations reported here were obtained primarily at the Las Campanas Observatory, a facility of the Observatories of the Carnegie Institution of Washington.

${ }^{2}$ Senior Visiting Fellow, Institute of Astronomy, 1 July 1990-1 August 1991.

${ }^{3}$ Current address: Steward Observatory, University of Arizona. of the confirmed 200 QSOs and 3 AGNs. In Sec. 3 we compare our sample with all previously detected QSOs in the three fields and update the redshift distribution for the published LBQS.

\section{OBSERVATIONS}

\subsection{Plate Material and Candidate Selection}

Three pairs of direct and objective-prism United Kingdom Schmidt Telescope (UKST) plates, details of which are presented in Table 1, were searched for QSO candidates. The Automated Plate Measuring facility scans an area of $\sim 5.8^{\circ} \times 5.8^{\circ}$ on each plate, but the exclusion of areas around bright sources and near calibration wedges from further processing reduces the total area for the present fields to $94.9^{\circ}$ sq. Furthermore, approximately $10 \%$ of the spectra cannot be processed because of plate flaws, satellite trails, or because they overlap with those of nearby sources. This results in an effective area of $85.5^{\circ}$ sq., the figure that should be used for surface density and luminosity function calculations. Candidates fainter than $B_{J}=16.0$ and brighter than the adopted limiting magnitude for each field (Table 1) were observed, irrespective of their direct image morphology. Candidate selection procedures have been described in $\mathbf{P a}$ pers II and III. 
TABLE 1. Plate data. ${ }^{\mathrm{a}}$

\begin{tabular}{cccccccccc}
\hline \hline Plate No. & R.A. (1950) Dec. & Filter & Exp. Time $^{b}$ & Date & \multicolumn{2}{c}{ Grade $^{c}$ Secing B $_{\text {Jie }}$} \\
\hline J9766 & 0053 & -2800 & GG395 & 70 & 23 Nov 1984 & A & 2.5 & 18.76 \\
UJ3682P & 0053 & -2800 & none & 70 & 17 Oct. 1977 & A1 & 2.0 & \\
J6229 & 2128 & -4500 & GG395 & 65 & 09 Aug 1980 & A & 2.0 & 18.55 \\
UJ4520P & 2128 & -4500 & none & 60 & 30 Sept. 1978 & AI3 & 2.5 & \\
J1746 & 2203 & -1855 & GG395 & 70 & 06 Aug 1975 & BI4 & 4.0 & 18.46 \\
UJ6526P & 2203 & -1855 & none & 45 & 30 Oct 1980 & A & 2.0 & \\
& & & & & & & & & \\
\hline \hline
\end{tabular}

Notes to TABLE 1

a All plates are Kodak IIIa-J emulsion.

${ }^{b}$ Exposure times in minutes.

c Details of the UKST grading system can be found in the UKST Handbook (1983).

\subsection{Magnitude Calibration}

Instrumental $B_{J}$ magnitudes for the three UKST direct III $a$-J plates were calibrated using a photometric sequence established from CCD $B$ and $V$ frames in five areas in each field obtained with the Las Campanas $1 \mathrm{~m}$ telescope. Further details on photometric calibration of the LBQS may be found in Paper II.

\subsection{Spectroscopic Observations and Redshift Determinations}

All but four of the spectra reported in this paper were obtained with the Las Campanas $2.5 \mathrm{~m}$ duPont telescope, using the Boller and Chivens spectrograph. The 2D-Frutti system which records the spectrum consists of a continuously readout Fairchild $380 \times 244$ CCD behind an image intensifier chain. Real-time processing centroids individual photon events to better than $1 / 2$ a CCD pixel, and we used a $64 \times 3040$ output pixel format. The observations were obtained in three observing runs: 22 August 1987-30 August 1987, 8 September-12 September 1988, and 28 August 1989-1 September 1989. A $600 \mathrm{~g} / \mathrm{mm}$ grating was used for the first run, and a $300 \mathrm{~g} / \mathrm{mm}$ grating for the remaining two. The 2D-Frutti-300 g/mm grating combination yields approximately $2.7 \AA$ and 1.4 arcsec per output pixel in the wavelength and spatial directions, respectively. We used a 90-arcsec-long slit with the width varying between 1.6 and 2.0 arcsec. The $300 \mathrm{~g} / \mathrm{mm}$ grating and a 2.0 arcsec slit gives a resolution (FWHM) of 4 pixels or $11 \AA$, with useful coverage from $3200 \AA$ to approximately $7500 \AA$, (second-order light contaminates the data longward of $6400 \AA$ ). The 600 $\mathrm{g} / \mathrm{mm}$ grating gives twice the resolution but somewhat less useful coverage at the red end.

During each night observations were made of two to three standard stars, and arc spectra were obtained at regular intervals. Because of the rather severe distortions caused by the intensifier, subtraction of the strongest night sky lines was not perfect and many of the spectra contain residual features at the positions of the strongest lines, notably [ $\mathrm{O}_{\mathrm{I}}$ ] $\lambda 5577$ and $\lambda 6300$. Data reduction consisted of flatfielding, wavelength calibration (using a 4th order polynomial fit to the arc spectra), sky subtraction (using a fit to two 15 arcsec windows separated by 10 arcsec from the object on either side), and flux calibration. Due to variable seeing and occasional cloud, the absolute flux calibration is uncertain for some objects.
Two QSOs (2128 - 4555 and 2129 - 4307) were observed at the AAT in September 1989 using the RGO spectrograph and IPCS. These observations spanned the wavelength range from 3850 to $5828 \AA$. No standard stars were observed so that the spectra are presented in units of detected photons. Finally, two QSOs were observed at CTIO using the RC Spectrograph. In October 1989, $2154-2105$ was observed over the range $3200-7000 \AA$ at a resolution of about $10 \AA$ using the 2D-Frutti. In October 1990, $2154-2005$ was observed at comparable resolution over the wavelength range 3500-6900 A using a UV-flooded TI CCD on the Folded Schmidt Camera.

Of the 300 candidates in the three new fields presented here, 200 were found to be QSOs. All 182 QSOs with magnitudes within the LBQS limits (Table 1) are listed in Table 2 in order of right ascension. Column 1 gives the name, columns 2 and 3 the celestial coordinates (equinox 1950.0), column 4 the emission-line redshift, column 5 the $B_{J}$ magnitude, column 6 the UT date of the spectroscopic observation, and column 7 comments on the spectrum. As in previous papers we estimate our coordinate and magnitude accuracy to be \pm 1 arcsec and \pm 0.15 mag, respectively. Redshifts were derived using the composite spectrum of $718 \mathrm{LBQS}$ QSOs (Francis et al. 1991) as a template against which to cross correlate the spectrum of each confirmed QSO.

Figure 1 presents flux-calibrated spectra of all objects listed in Table 2. Except for the two QSOs noted above observed at the AAT, the $y$ axis represents the flux per unit wavelength, but the scale factors are arbitrary; for the AAT spectra the raw counts are plotted.

Figure 2 and Table 3 present the same information as contained in Fig. 1 and Table 2 for confirmed QSOs that are fainter than the LBQS limit in their corresponding field. They should not be considered part of the LBQS sample.

Figure 3 and Table 4 present spectra and relevant parameters, respectively, for those extragalactic objects with $z \geqslant 0.2$ and $M_{B_{J}}>-21.5$, the adopted absolute magnitude threshold for the LBQS sample. In computing the absolute magnitude of the objects, a Hubble constant of $100 \mathrm{~km} \mathrm{~s}^{-1} \mathrm{Mpc}^{-1}$, $q_{0}=0.0$ and $k$ corrections assuming the QSOs are represented by a simple power-law flux distribution $F(v) \propto v^{\alpha}$, with $\alpha=-1$ have been adopted.

Table 5 presents relevant parameters for extragalactic objects with $z<0.2$, the adopted redshift threshold for the LBQS sample.

\section{COMPARISON WITH PREVIOUSLY KNOWN QSOS}

As in Papers I-IV, we compare our list of QSOs with previously published lists as a crude test of our selection efficiency. We rely primarily on the Hewitt-Burbidge (HB) catalogue $(1987,1989)$ for this comparison, although two recent studies by Boyle et al. (1990) and Cristiani et al. (1990) are also included in our search since they report additional QSOs in the fields presented here. We restrict our comparison to objects with $z>0.2$ and published magnitudes between 16.0 and 19.0, irrespective of the optical passband employed. We also include objects for which no magnitude is given in $\mathrm{HB}$.

Of the 131 QSOs meeting our redshift and magnitude criteria listed in the above references, our procedures have independently identified 62 . The remaining 69 are listed in Table 6 , where the coordinates given are those measured from our UKST direct plates. Unless otherwise noted, the entries are taken from HB. Sixty-one of the objects are fainter than our 
TABLE 2. Confirmed QSOS.

\begin{tabular}{|c|c|c|c|c|c|c|c|c|c|c|c|c|c|}
\hline Designation & R.A. (1950) & Dec. $(1950)$ & $\mathrm{z}_{\mathrm{em}}$ & $\mathrm{B}_{\mathbf{J}}$ & UT Date & Comment & Designation & R.A. (1950) & Dec. $(1950)$ & $\mathrm{z}_{\mathrm{em}}$ & $\mathrm{B}_{\mathbf{J}}$ & UT Date & Comment \\
\hline $0039-2630$ & 003936.3 & -263032 & 1.810 & 17.3 & $08 / 30 / 89$ & Hв & $0051-2605$ & 005153.5 & -260517 & 0.624 & 18.3 & $09 / 10 / 88$ & \\
\hline $0039-2727$ & 003950.0 & -272729 & 1.407 & 18.6 & $08 / 29 / 89$ & & $0052-3020$ & 005200.7 & -302052 & 0.990 & 17.7 & $08 / 28 / 89$ & f \\
\hline $0040-3024$ & 004033.2 & -302408 & 0.609 & 18.0 & $08 / 30 / 87$ & & $0052-2945$ & 005226.9 & -294549 & 0.760 & 18.7 & $09 / 08 / 88$ & \\
\hline $0040-2917$ & 004041.4 & -291722 & 2.087 & 17.8 & $08 / 29 / 89$ & & $0052-2856$ & 005240.9 & -285648 & 0.602 & 18.3 & $08 / 31 / 89$ & $o, c$ \\
\hline $0040-2919$ & 004046.3 & -291940 & 0.624 & 18.4 & $08 / 24 / 87$ & $o, c$ & $0052-2550$ & 005241.2 & -255039 & 0.855 & 18.6 & $09 / 11 / 88$ & \\
\hline $0041-2638$ & 004115.2 & -263836 & 3.053 & 18.3 & $08 / 30 / 89$ & & $0052-3108$ & 005250.8 & -310833 & 0.350 & 18.1 & $08 / 30 / 87$ & \\
\hline $0041-2844$ & 004124.2 & -284407 & 0.839 & 18.3 & $08 / 29 / 87$ & & $0052-2853 B$ & 005251.4 & -285335 & 0.634 & 18.6 & $08 / 28 / 89$ & $o, c$ \\
\hline $0041-2707$ & 004124.4 & -270754 & 2.786 & 18.0 & $09 / 12 / 88$ & $\mathrm{HB}$ & $0053-2639$ & 005311.3 & -263902 & 0.808 & 18.6 & $09 / 08 / 88$ & \\
\hline $0041-2904$ & 004128.6 & -290416 & 0.674 & 17.9 & $09 / 10 / 88$ & & $0053-2709$ & 005338.6 & -270911 & 1.039 & 18.2 & $08 / 28 / 87$ & HB \\
\hline $0041-2607$ & 004131.1 & -260742 & 2.505 & 17.1 & $08 / 30 / 89$ & $\mathrm{HB}$ & $0053-2526$ & 005339.3 & -252623 & 1.379 & 18.4 & $08 / 29 / 87$ & \\
\hline $0041-2658$ & 004138.4 & -265830 & 2.457 & 18.6 & $08 / 28 / 89$ & & $0053-2813$ & 005347.2 & -281325 & 0.725 & 18.5 & $08 / 26 / 87$ & \\
\hline $0041-2859$ & 004140.9 & -285936 & 2.134 & 18.0 & $08 / 29 / 87$ & & $0054-3101$ & 005427.6 & -310101 & 1.788 & 18.7 & $08 / 29 / 89$ & \\
\hline $0042-2627$ & 004206.4 & -262746 & 3.289 & 18.5 & $08 / 29 / 89$ & HB, L & $0055-2744$ & 005509.7 & -274440 & 2.195 & 18.6 & $09 / 11 / 88$ & HВ \\
\hline $0042-2908$ & 004207.5 & -290815 & 1.250 & 18.4 & $08 / 29 / 87$ & & $0055-2559$ & 005514.3 & -255906 & 0.582 & 18.2 & $08 / 28 / 89$ & HB,n \\
\hline $0042-2550$ & 004217.1 & -255037 & 0.454 & 18.3 & $08 / 24 / 87$ & $o, c$ & $0055-2948$ & 005543.3 & -294859 & 0.663 & 18.5 & $08 / 28 / 89$ & $\mathrm{HB}$ \\
\hline $0042-2750$ & 004226.9 & -275021 & 0.741 & 18.2 & $08 / 26 / 87$ & & $0056-2915$ & 005620.8 & -291534 & 1.257 & 18.5 & $08 / 28 / 89$ & $\mathrm{HB}$ \\
\hline $0042-3053$ & 004235.7 & -305328 & 1.974 & 18.5 & $08 / 30 / 89$ & & $0056-2843$ & 005641.3 & $-2843 \quad 14$ & 0.934 & 17.8 & $08 / 24 / 87$ & \\
\hline $0042-2930$ & 004241.9 & -293058 & 2.388 & 17.8 & $08 / 29 / 87$ & $2 \mathrm{~d}, 1 \mathrm{~d} ?$ & $0056-2948$ & 005658.1 & -294819 & 0.351 & 18.4 & $08 / 25 / 87$ & \\
\hline $0042-2657$ & 004252.3 & -265716 & 2.898 & 18.7 & $09 / 11 / 88$ & $\mathrm{n}$ & $0057-2630$ & 005730.2 & -263023 & 1.042 & 18.6 & $09 / 09 / 88$ & \\
\hline $0042-2729$ & 004255.3 & -272907 & 0.933 & 18.7 & $09 / 09 / 88$ & & $0057-2908$ & 005740.6 & -290815 & 0.489 & 18.4 & $08 / 25 / 87$ & $\mathrm{HB}, \mathrm{n}, \mathrm{o}, \mathrm{c}$ \\
\hline $0043-2743$ & 004322.6 & -274334 & 1.049 & 18.5 & $08 / 26 / 87$ & & $0057-2729$ & 005756.6 & -272946 & 1.203 & 18.5 & $08 / 28 / 89$ & $\mathrm{HB}$ \\
\hline $0043-2937$ & 004302.1 & -293702 & 2.230 & 18.8 & $09 / 10 / 88$ & & $0058-2604$ & 005806.2 & -260445 & 2.472 & 18.5 & $08 / 24 / 87$ & \\
\hline $0043-3006$ & 004349.8 & -300634 & 1.124 & 18.7 & $09 / 01 / 89$ & & $0058-2919$ & 005834.5 & -291901 & 1.193 & 18.4 & $08 / 28 / 89$ & $\mathrm{HB}$ \\
\hline 0045-3002 & 004504.7 & -300253 & 2.021 & 18.3 & $09 / 12 / 88$ & $\mathrm{HB}$ & $0058-2907$ & 005835.6 & -290723 & 0.866 & 18.4 & $08 / 25 / 87$ & \\
\hline 0045-3045 & 004514.4 & -304501 & 0.979 & 17.4 & $08 / 30 / 89$ & & $0059-2525$ & 005914.9 & -252506 & 0.454 & 17.6 & $08 / 31 / 89$ & a \\
\hline $0045-2806$ & 004541.6 & -280623 & 1.138 & 18.7 & $09 / 11 / 88$ & & $0059-2545$ & 005921.4 & -254559 & 1.955 & 18.5 & $09 / 01 / 89$ & \\
\hline $0045-2606$ & 004545.4 & -260627 & 1.242 & 18.0 & $08 / 29 / 89$ & $\mathrm{~b} ?$ & $0059-2625$ & 005927.7 & -262508 & 2.100 & 18.6 & $09 / 11 / 88$ & $\mathrm{HB}$ \\
\hline $0046-2812$ & 004601.8 & $-28 \quad 1240$ & 1.689 & 18.3 & $09 / 12 / 88$ & $\mathrm{HB}, \mathrm{n}$ & $0059-3034$ & 005937.1 & -303434 & 1.033 & 17.2 & $08 / 24 / 87$ & \\
\hline $0046-2834$ & 004618.0 & -283401 & 0.634 & 17.7 & $09 / 01 / 89$ & $\mathrm{HB}, \mathrm{o}, \mathrm{c}$ & $0059-2946$ & 005950.3 & -294619 & 1.076 & 17.8 & $08 / 28 / 89$ & \\
\hline $0046-2914$ & 004650.7 & -291440 & 0.781 & 18.0 & $09 / 09 / 88$ & & $0059-2735$ & 005952.4 & -273557 & 1.600 & 18.1 & $09 / 12 / 88$ & HB, $\mathbf{b}^{\prime}$ \\
\hline $0047-3059$ & 004708.9 & -305951 & 0.559 & 18.0 & $08 / 30 / 87$ & $\mathrm{o}, \mathrm{c}$ & $0100-2809$ & 010027.5 & -280910 & 1.768 & 18.2 & $08 / 25 / 87$ & a \\
\hline $0047-2759$ & 004749.7 & -275936 & 2.130 & 18.3 & $08 / 30 / 87$ & $\mathrm{HB}$ & $0100-2702$ & 010031.6 & -270243 & 1.606 & 17.9 & $08 / 28 / 89$ & $\mathrm{HBr}$ \\
\hline $0047-2522$ & 004752.1 & -252232 & 1.184 & 18.7 & $08 / 30 / 89$ & & $0100-3105$ & $\begin{array}{llll}01 & 00 & 39.7\end{array}$ & -310544 & 2.641 & 18.3 & $08 / 30 / 87$ & $\mathrm{n}, \mathrm{d}$ \\
\hline $0047-2647 \mathrm{~B}$ & 004754.3 & -264755 & 0.495 & 18.6 & $08 / 28 / 89$ & $\mathrm{HB}$ & 0101-2708 & 010101.8 & -270850 & 0.558 & 18.6 & $09 / 10 / 88$ & \\
\hline $0047-3050$ & 004754.7 & -305041 & 2.973 & 18.5 & $08 / 29 / 89$ & L? & $0101-2548$ & 010108.2 & -254833 & 1.973 & 18.6 & $08 / 30 / 87$ & \\
\hline $0047-2538$ & 004757.9 & -253856 & 1.969 & 18.4 & $08 / 29 / 87$ & & $0101-2611$ & 010123.9 & -261105 & 0.277 & 18.0 & $08 / 29 / 89$ & \\
\hline $0048-2901$ & 004824.2 & -290146 & 0.783 & $18: 3$ & $08 / 24 / 87$ & & 0101-3025 & 010132.0 & -302552 & 3.152 & 18.5 & $08 / 29 / 89$ & $\mathrm{HB}, \mathrm{L}$ \\
\hline $0048-2918$ & 004826.4 & -291815 & 0.435 & 18.3 & $08 / 30 / 89$ & & $0102-2713$ & 010216.6 & -271312 & 0.780 & 17.5 & $08 / 29 / 89$ & $\mathrm{HB}, \mathrm{f}$ \\
\hline $0048-2545$ & 004835.4 & -254508 & 2.082 & 18.2 & $09 / 20 / 88$ & & $0102-2634$ & 010256.6 & -263418 & 1.223 & 18.3 & $08 / 29 / 89$ & нВ \\
\hline $0048-2804$ & 004847.1 & -280419 & 0.845 & 17.9 & $08 / 27 / 87$ & $\mathrm{HB}$ & 0103-2753 & $\begin{array}{lll}01 & 03 & 10.7\end{array}$ & -275302 & 0.848 & 18.1 & $08 / 26 / 87$ & $b, f$ \\
\hline $0049-2820$ & 004901.2 & -282053 & 2.256 & 18.4 & $08 / 25 / 87$ & $\mathrm{HB}, 2 \mathrm{~d}$ & $0103-2901$ & 010333.0 & -290132 & 2.870 & 18.5 & $08 / 28 / 87$ & $\mathrm{~d} ?$ \\
\hline $0049-2840$ & 004942.3 & -284027 & 0.640 & 18.7 & $09 / 08 / 88$ & $o, c$ & $0103-2622$ & 010334.3 & -262224 & 0.776 & 16.9 & $08 / 30 / 89$ & \\
\hline $0049-2535$ & 004944.3 & -253517 & 1.528 & 18.5 & $09 / 10 / 88$ & $\mathrm{~b} ?$ & $0103-2913$ & 010354.3 & -291305 & 2.803 & 18.5 & $09 / 12 / 88$ & $\mathrm{HB}, \mathrm{L}$ \\
\hline $0049-3050$ & 004944.4 & -305013 & 0.637 & 18.5 & $09 / 10 / 88$ & $o, c$ & $0103-2749$ & 010357.3 & -274952 & 1.018 & 18.3 & $08 / 28 / 87$ & \\
\hline $0050-2905$ & 005005.9 & -290536 & 1.610 & 18.5 & $09 / 12 / 88$ & HB, $n$ & $0104-2809$ & 010415.2 & -280957 & 1.584 & 18.4 & $08 / 28 / 87$ & $\mathrm{HB}$ \\
\hline $0050-2523$ & 005018.0 & -252311 & 2.159 & 17.8 & $08 / 24 / 87$ & HB & $0105-2649$ & $0105 \quad 17.8$ & -264930 & 2.463 & 17.7 & $08 / 30 / 89$ & $\mathrm{n}$ \\
\hline $0050-2527$ & 005041.4 & -252739 & 1.019 & 17.6 & $08 / 28 / 89$ & $\mathrm{n}$ & $2110-4509$ & 211058.5 & -450905 & 0.555 & 18.5 & $09 / 11 / 88$ & $o, c$ \\
\hline $0050-2806$ & 005049.2 & -280629 & 1.823 & 18.7 & $09 / 12 / 88$ & HB & $2111-4506$ & $\begin{array}{lll}21 & 11 & 09.7\end{array}$ & -450650 & 1.376 & 17.3 & $09 / 11 / 88$ & \\
\hline $0050-2742$ & 005050.9 & -274206 & 0.479 & 17.9 & $08 / 27 / 87$ & HB o,c & $2111-4335$ & 211150.9 & -433539 & 1.708 & 16.7 & $08 / 22 / 87$ & b \\
\hline $0051-2839$ & 005121.7 & -283958 & 1.577 & 18.3 & $08 / 27 / 87$ & HВ & $2113-4305$ & 211323.7 & -430532 & 1.249 & 18.0 & $09 / 09 / 88$ & \\
\hline
\end{tabular}


TABLE 2. (continued)

\begin{tabular}{|c|c|c|c|c|c|c|c|c|c|c|c|c|c|}
\hline Designation & R.A. (1950) & Dec. $(1950)$ & $z_{\text {em }}$ & $\mathbf{B}_{\mathbf{J}}$ & UT Date & Comment & Designation & R.A. (1950) & Dec. $(1950)$ & $z_{e m}$ & $\mathbf{B}_{\mathbf{J}}$ & UT Date & Comment \\
\hline $2113-4538$ & 211325.5 & -453845 & 0.946 & 17.3 & $09 / 10 / 88$ & & $2154-2005$ & 215419.6 & -200533 & 2.040 & 18.1 & $10 / 23 / 90$ & HB \\
\hline $2113-4345$ & 211338.4 & -434508 & 2.053 & 18.5 & $09 / 11 / 88$ & a? & $2154-1908$ & 215438.0 & -190844 & 1.656 & 18.0 & $09 / 01 / 89$ & HB \\
\hline $2114-4346$ & 211403.5 & -434660 & 2.041 & 18.3 & $08 / 30 / 87$ & & $2154-2105$ & 215438.1 & -210526 & 0.570 & 17.6 & $10 / 08 / 89$ & $o, c$ \\
\hline $2114-4335$ & 211410.8 & -433534 & 1.318 & 17.9 & $09 / 11 / 88$ & & $2156-1819$ & 215604.7 & -181914 & 0.360 & 18.4 & $09 / 09 / 88$ & o \\
\hline $2115-4501$ & 211542.8 & -450159 & 0.597 & 18.3 & $08 / 27 / 87$ & & $2158-1855$ & 215814.3 & -185549 & 0.687 & 17.6 & $09 / 11 / 88$ & HB \\
\hline $2116-4439$ & 211655.2 & -443938 & 1.480 & 17.7 & $09 / 09 / 88$ & b & $2158-1657$ & 215850.0 & -165728 & 0.475 & 18.4 & $09 / 11 / 88$ & HB \\
\hline $2118-4702$ & 211829.2 & -470233 & 1.332 & 18.4 & $08 / 28 / 87$ & HBv & $2159-1906$ & 215905.8 & -190622 & 0.364 & 18.2 & $08 / 22 / 87$ & $\mathbf{n}$ \\
\hline $2119-4758$ & 211925.6 & -475855 & 1.464 & 18.4 & $09 / 12 / 88$ & & $2159-1926$ & 215924.0 & -192621 & 1.174 & 18.2 & $09 / 12 / 88$ & HB \\
\hline $2119-4415$ & 211925.7 & -441549 & 0.728 & 18.3 & $09 / 01 / 89$ & $\mathbf{n}$ & $2159-2058$ & 215948.7 & -205855 & 2.120 & 18.4 & $09 / 10 / 88$ & $\mathrm{a} ?$ \\
\hline $2119-4249$ & 211926.3 & -424941 & 1.054 & 18.3 & $09 / 08 / 88$ & $\mathrm{HBv}$ & $2200-2019$ & 220032.1 & -201952 & 0.671 & 18.4 & $09 / 11 / 88$ & \\
\hline $2120-4727$ & 212050.8 & -472716 & 1.591 & 18.0 & $08 / 29 / 87$ & & $2200-1759$ & 220039.2 & -175917 & 0.988 & 18.4 & $08 / 22 / 87$ & HB \\
\hline $2120-4327$ & 212050.9 & -432757 & 1.240 & 18.2 & $08 / 27 / 87$ & & $2200-1958$ & 220054.5 & -195821 & 1.260 & 17.9 & $08 / 22 / 87$ & $\mathbf{n}$ \\
\hline $2121-4406$ & 212142.0 & -44.0658 & 1.735 & 18.0 & $08 / 26 / 87$ & $\mathbf{n}$ & $2201-1834$ & 220116.9 & -183417 & 1.814 & 17.8 & $08 / 25 / 87$ & $\mathrm{HB}, \mathrm{b}$ ? \\
\hline $2122-4231$ & 212200.2 & -423144 & 2.266 & 18.1 & $09 / 09 / 88$ & & $2203-2145$ & 220304.8 & -214513 & 2.273 & 18.4 & $09 / 01 / 89$ & \\
\hline $2123-4338$ & 212321.3 & -433830 & 0.480 & 18.3 & $09 / 01 / 89$ & $0, c$ & $2203-2134$ & 220355.0 & -213422 & 0.576 & 18.2 & $09 / 01 / 89$ & $o, c$ \\
\hline $2127-4242$ & 212719.3 & -424246 & 0.799 & 17.7 & $08 / 27 / 87$ & & $2203-1833$ & 220355.2 & -183326 & 2.728 & 18.4 & $09 / 11 / 88$ & L? \\
\hline $2128-4327$ & 212813.1 & -432707 & 0.920 & 17.4 & $08 / 26 / 87$ & & $2204-1911$ & 220432.2 & -191141 & 1.063 & 17.6 & $08 / 26 / 87$ & HB \\
\hline $2128-4555$ & 212813.8 & -455507 & 0.624 & 18.4 & $09 / 13 / 89$ & $\mathrm{U}, \mathrm{o}$ & $2204-2043$ & 220449.7 & -204353 & 1.689 & 17.9 & $09 / 09 / 88$ & \\
\hline $2128-4610$ & 212821.9 & -461052 & 0.833 & 17.6 & $08 / 28 / 87$ & HBv & $2205-2014$ & 220518.5 & -201441 & 2.635 & 18.4 & $09 / 08 / 88$ & $d ?$ \\
\hline $2129-4307$ & 212905.3 & -430728 & 0.311 & 18.4 & $09 / 13 / 89$ & $\mathrm{U}$ & $2205-2053$ & 220521.4 & -205338 & 1.546 & 18.1 & $09 / 09 / 88$ & HB \\
\hline $2129-4624$ & 212939.4 & -462419 & 0.435 & 17.9 & $08 / 25 / 87$ & a? & $2205-2129$ & 220525.2 & -212948 & 1.420 & 18.5 & $09 / 12 / 88$ & \\
\hline $2131-4257$ & 213133.3 & -425752 & 2.096 & 18.3 & $08 / 27 / 87$ & $\mathrm{HBv}$ & $2205-2001$ & 220551.6 & -200131 & 1.716 & 17.9 & $09 / 01 / 89$ & HB \\
\hline $2132-4451$ & 213237.5 & -445102 & 0.920 & 18.5 & $09 / 08 / 88$ & $\mathrm{HBv}$ & $2205-1940$ & 220559.3 & -194015 & 1.280 & 17.9 & $08 / 27 / 87$ & HB \\
\hline $2132-4321$ & 213254.9 & -432145 & 2.420 & 18.2 & $08 / 26 / 87$ & & $2206-1958 \mathrm{~A}$ & 220606.9 & -195845 & 2.558 & 17.5 & $09 / 11 / 88$ & $\mathrm{HB}, 2 \mathrm{~d}$ \\
\hline $2132-4240$ & 213258.0 & -424007 & 1.629 & 18.5 & $08 / 29 / 87$ & & $2206-1803$ & 220640.9 & -180302 & 1.050 & 18.4 & $09 / 12 / 88$ & \\
\hline $2134-4239$ & 213438.1 & -423943 & 1.804 & 17.9 & $08 / 30 / 87$ & & $2207-1703$ & 220708.9 & -170341 & 0.371 & 18.4 & $09 / 10 / 88$ & o \\
\hline $2135-4243$ & 213514.3 & -424349 & 0.250 & 17.2 & $09 / 10 / 88$ & & $2207-2044$ & 220744.2 & -204427 & 1.168 & 18.1 & $08 / 26 / 87$ & \\
\hline $2135-4530$ & 213514.3 & -453050 & 0.308 & 17.7 & $09 / 09 / 88$ & & $2207-1627$ & 220751.8 & -162706 & 1.318 & 17.0 & $10 / 13 / 88$ & u \\
\hline $2135-4620$ & $\begin{array}{lll}21 & 35 & 19.3\end{array}$ & -462047 & 0.505 & 18.5 & $08 / 26 / 87$ & & $2208-1927$ & 220803.5 & -192738 & 1.532 & 18.2 & $09 / 12 / 88$ & HB,b?? \\
\hline $2135-4253$ & 213520.0 & -425330 & 1.469 & 18.4 & $08 / 29 / 87$ & $\mathrm{HBv}$ & $2208-1809$ & 220819.1 & -180947 & 1.290 & 17.7 & $08 / 25 / 87$ & \\
\hline $2136-4301$ & 213630.3 & -430123 & 1.343 & 18.2 & $08 / 29 / 87$ & & $2208-1720$ & 220832.1 & -172015 & 1.210 & 17.6 & $09 / 10 / 88$ & b \\
\hline $2136-4344$ & 213646.5 & -434438 & 0.490 & 18.2 & $09 / 10 / 88$ & & $2208-1905$ & 220852.5 & -190537 & 1.225 & 18.0 & $08 / 22 / 87$ & a? \\
\hline $2137-4435$ & 213751.5 & -443549 & 0.632 & 18.4 & $09 / 01 / 89$ & $o, c$ & $2209-1842$ & 220926.3 & -184230 & 2.092 & 17.7 & $09 / 01 / 89$ & $\mathrm{HB}, \mathrm{n}$ \\
\hline $2138-4430$ & 213818.4 & -443014 & 1.104 & 18.5 & $09 / 09 / 88$ & & $2209-2124$ & 220959.5 & -212433 & 1.714 & 18.3 & $09 / 01 / 89$ & \\
\hline $2138-4650$ & 213841.4 & -465032 & 0.762 & 18.5 & $08 / 28 / 87$ & & $2210-1751$ & 221026.2 & -175155 & 1.557 & 17.9 & $09 / 09 / 88$ & $\mathrm{~b} ? ?$ \\
\hline $2139-4303$ & 213954.7 & -430311 & 0.320 & 17.5 & $09 / 11 / 88$ & & $2211-1745$ & 221146.2 & -174506 & 0.905 & 18.2 & $09 / 11 / 88$ & \\
\hline $2140-4552$ & 214016.3 & -455237 & 1.688 & 18.3 & $08 / 27 / 87$ & $\mathrm{~b}$ ? & $2211-1915$ & 221153.7 & -191554 & 1.952 & 18.0 & $09 / 01 / 89$ & $\mathrm{HB}, \mathrm{b}$ ? \\
\hline $2142-4449$ & 214216.7 & -444932 & 1.025 & 18.2 & $09 / 10 / 88$ & & $2212-1634$ & 221213.2 & -163426 & 0.296 & 17.8 & $09 / 01 / 89$ & \\
\hline $2142-4318$ & 214242.7 & -431856 & 1.118 & 18.5 & $09 / 12 / 88$ & & $2212-1747$ & 221232.0 & -174720 & 1.159 & 18.3 & $09 / 01 / 89$ & \\
\hline \multirow[t]{3}{*}{$2153-2056$} & 215306.6 & -205603 & 1.849 & 17.8 & $09 / 01 / 89$ & HB & $2212-1759$ & 221248.3 & -175907 & 2.217 & 17.9 & $09 / 12 / 88$ & $\mathrm{HB}, \mathrm{b}$ \\
\hline & & & & & & & $2214-2048$ & 221406.6 & -204805 & 1.684 & 18.3 & $09 / 01 / 89$ & HB \\
\hline & & & & & & & $2214-1903$ & 221407.8 & -190314 & 0.396 & 18.2 & $09 / 01 / 89$ & \\
\hline
\end{tabular}

Notes to TABLE 2

a - 'Associated' absorption present, namely strong non-BAL absorption within $5000 \mathrm{kms}^{-1}$ of the corresponding emission line.

$\mathrm{b}$ - BAL QSO; b? - probable BAL QSO; b?? - possible BAL QSO

$c$ - If single line were CIII] $\lambda 1909$, C IV $\lambda 1550$ would be observable.

$\mathrm{d}$ - Damped Lyman $\alpha$ candidate.

$\mathrm{f}$ - Strong UV Fe II emission.

L - Lyman limit absorption.

n - Narrow emission lines.

o- One-line redshift.

$\mathrm{u}$ - Droop in UV may be due to atmospheric dispersion losses at large airmass.

U -- Uncalibrated flux scale.

$\mathrm{HB}$ - Tabulated in Hewitt and Burbidge (1987); $\mathrm{HBr}$ - radio-selected; HBv — Variability-selected. 


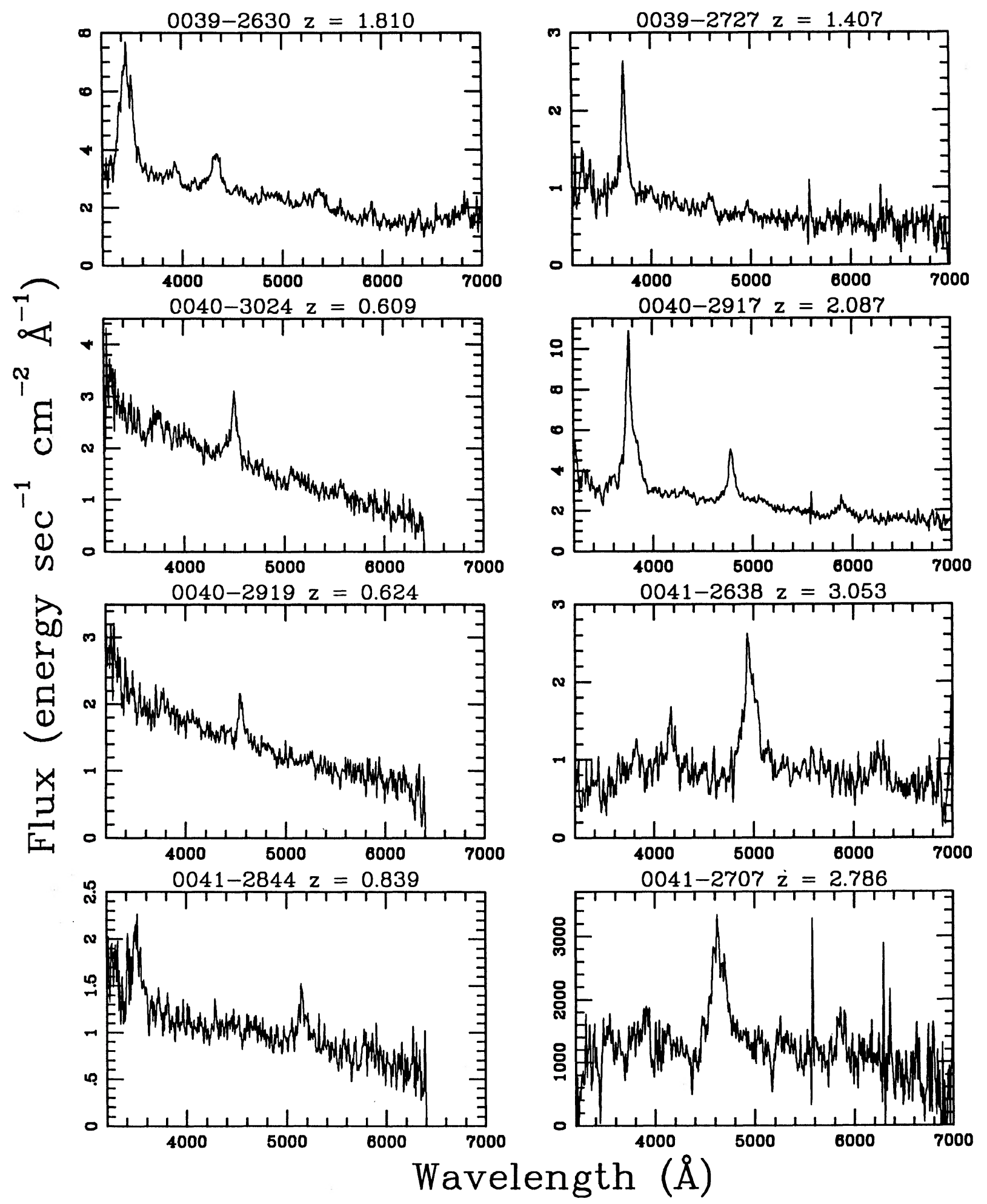

FIG. 1. Intermediate-resolution spectra, in order of right ascension, of LBQS QSOs. Except for $2128-4555$ and $2129-4307$, for which the ordinate is proportional to the raw counts, the flux per unit wavelength is plotted, but the flux scales are arbitrary. 


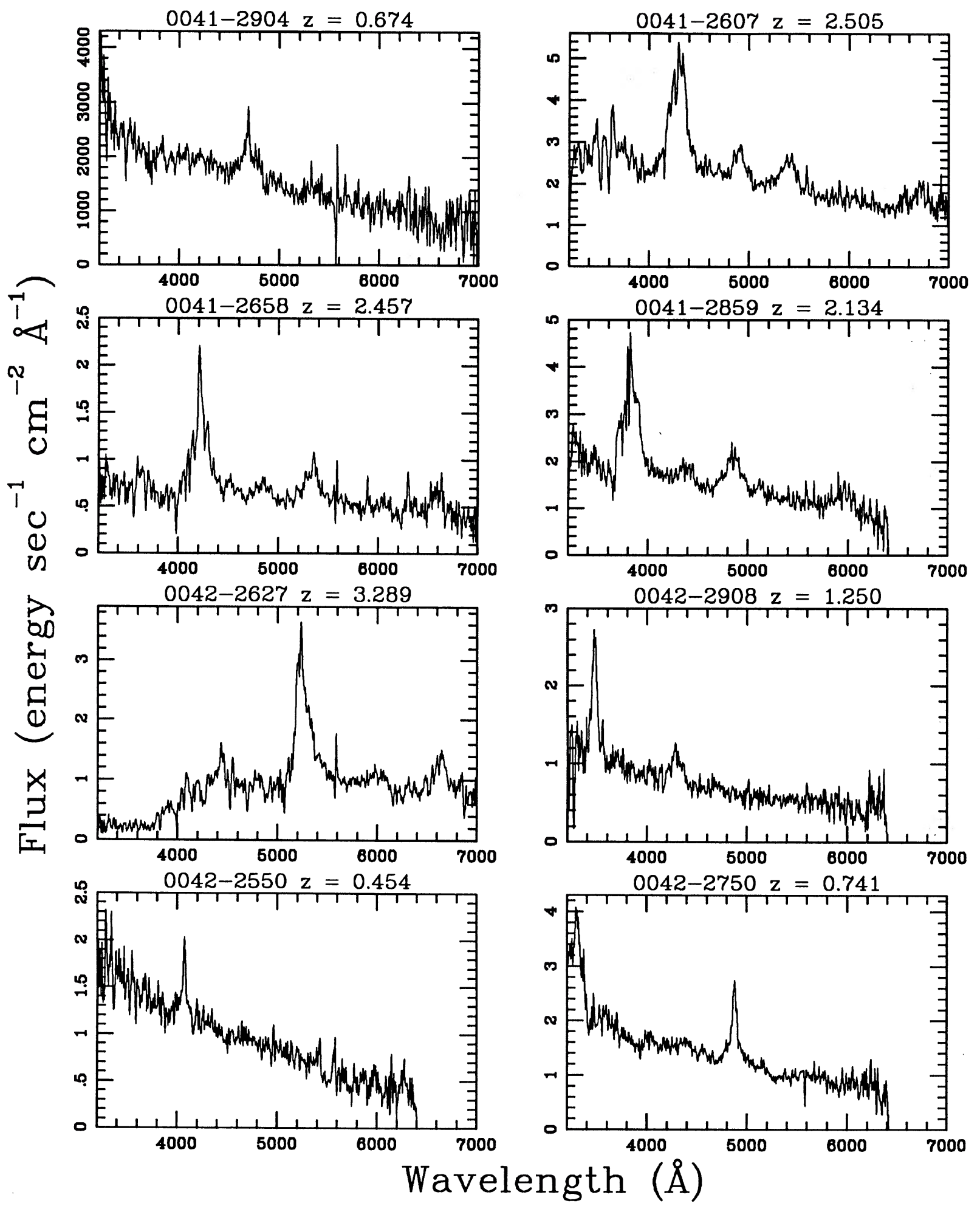

FIG. 1. (continued) 


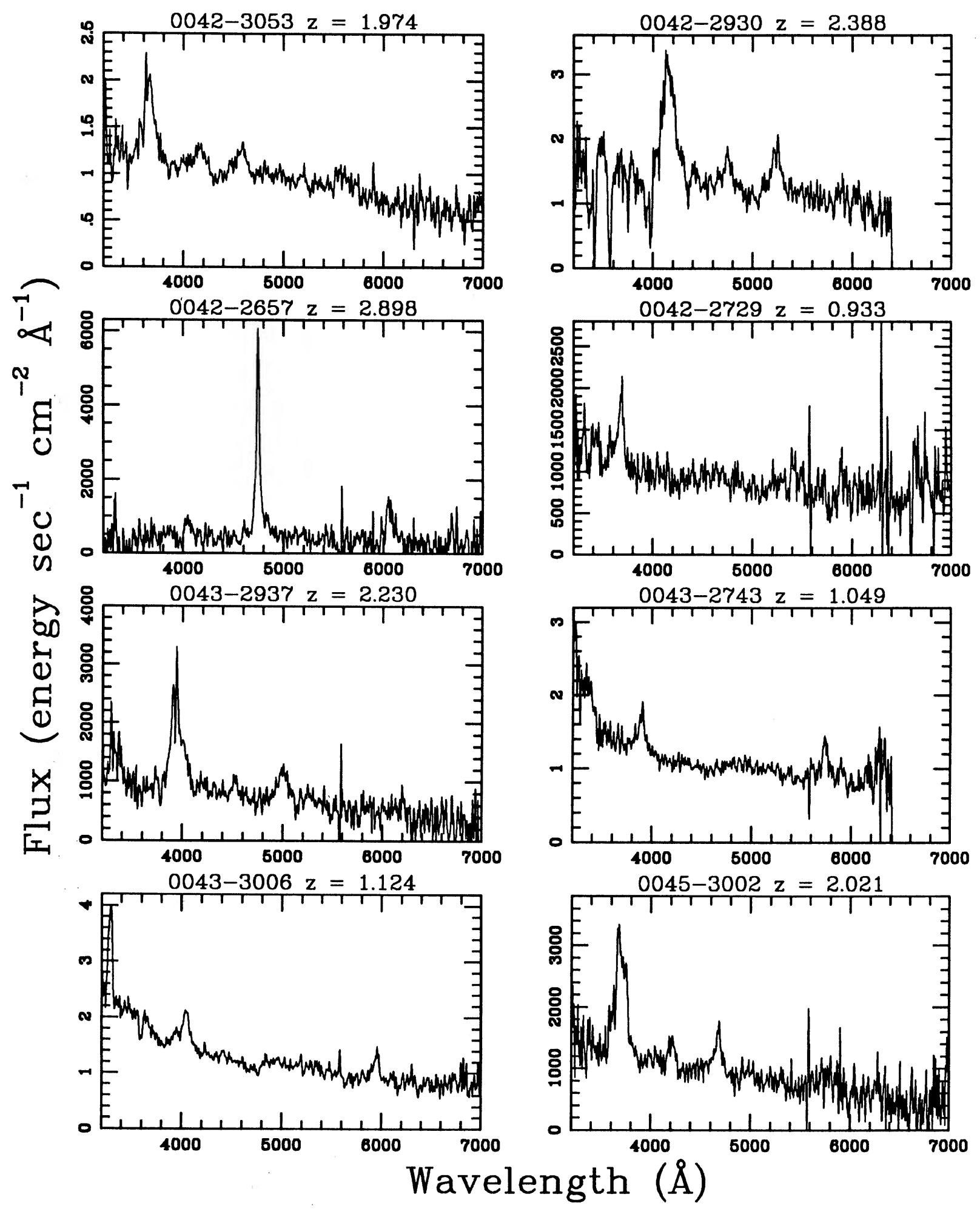

FIG. 1. (continued) 

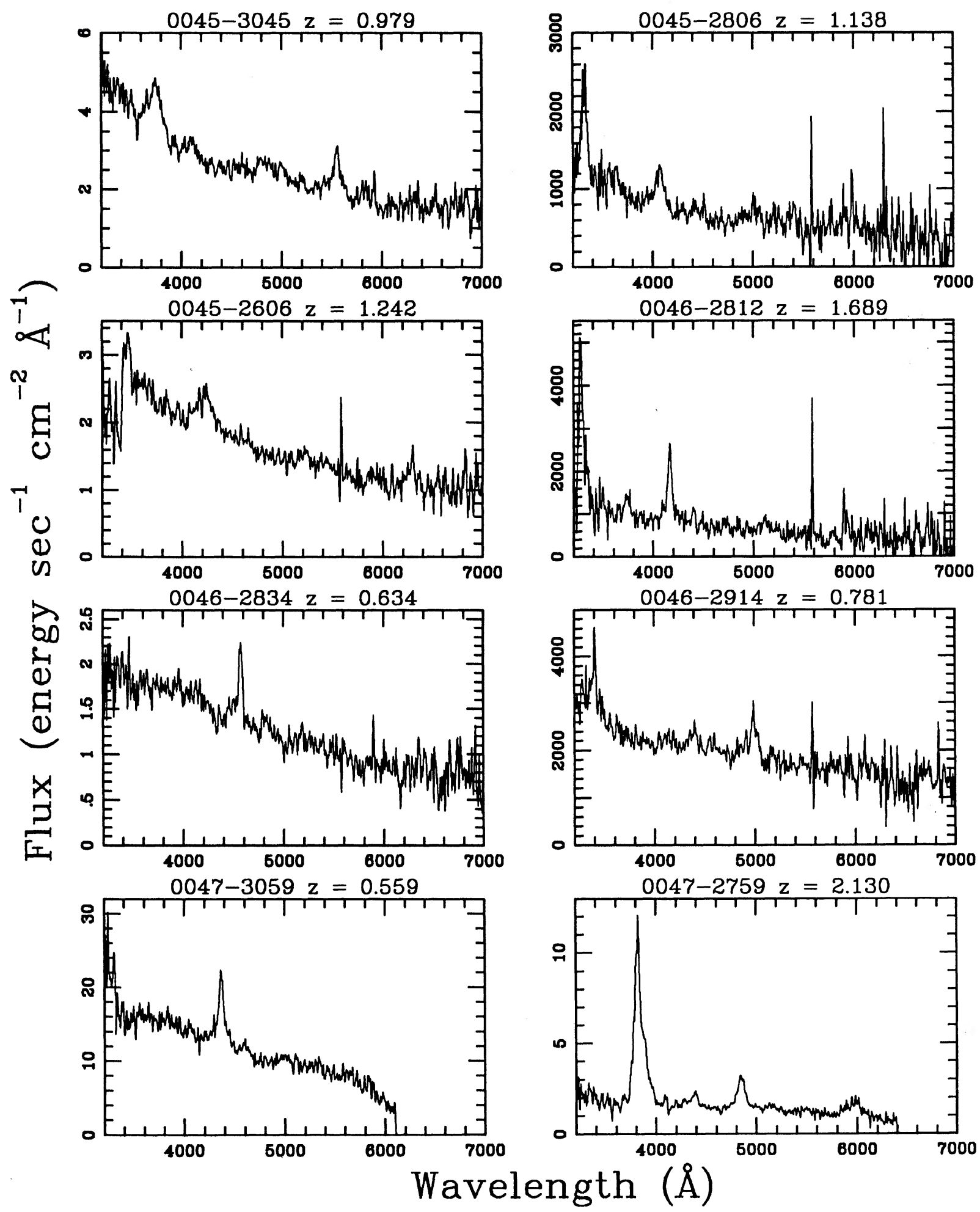

FIG. 1. (continued) 


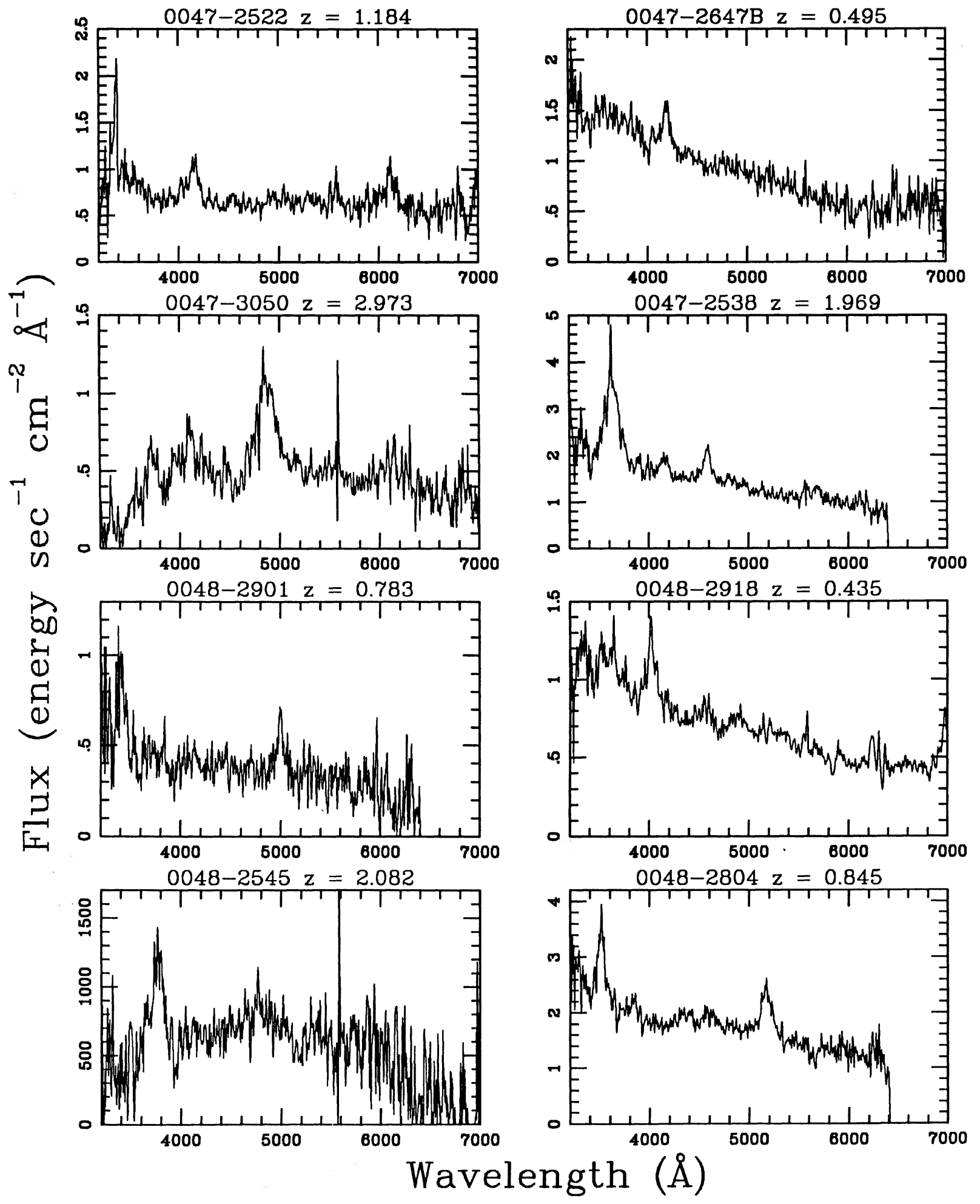

FIG. 1. (continued) 


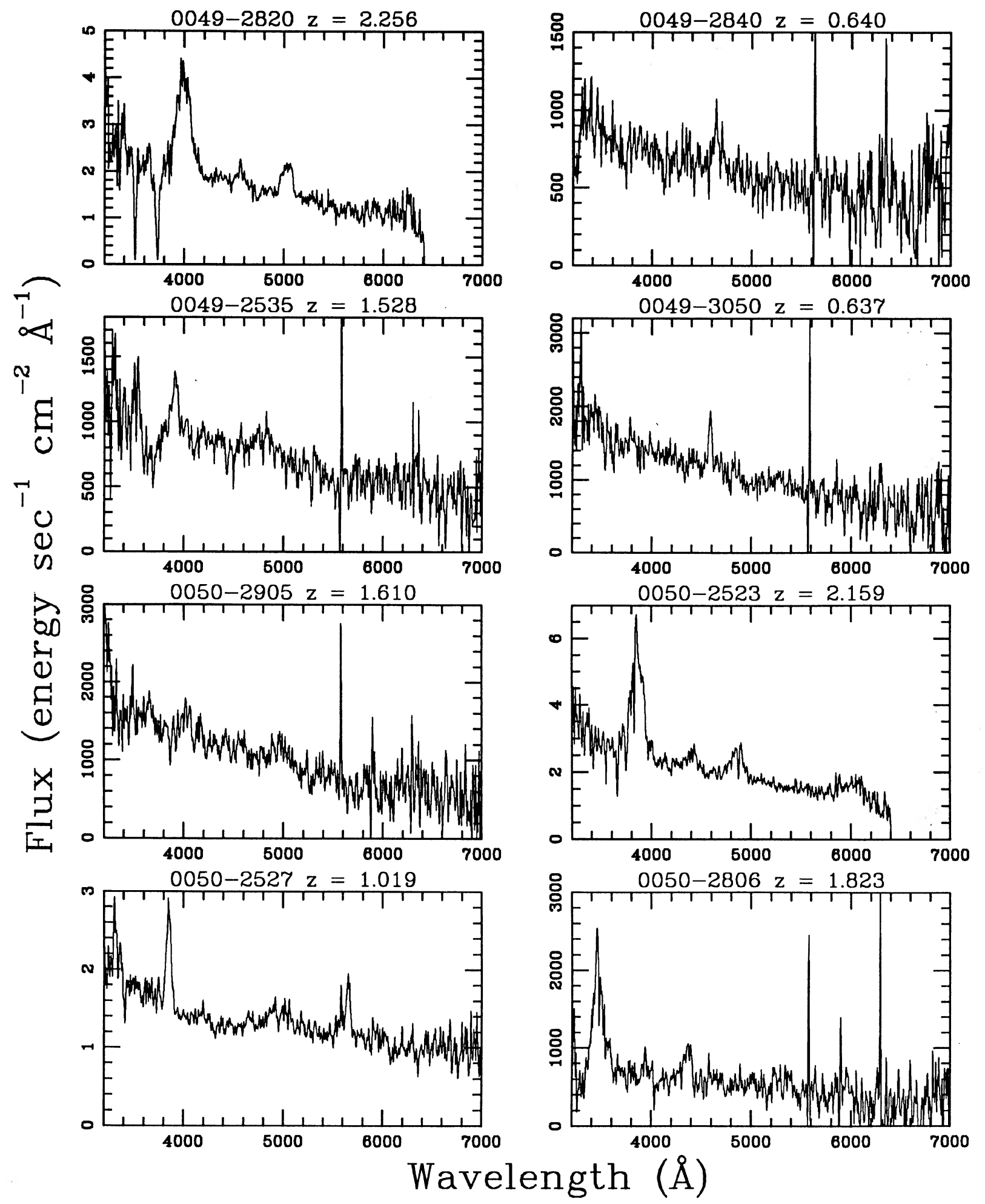

FIG. 1. (continued) 


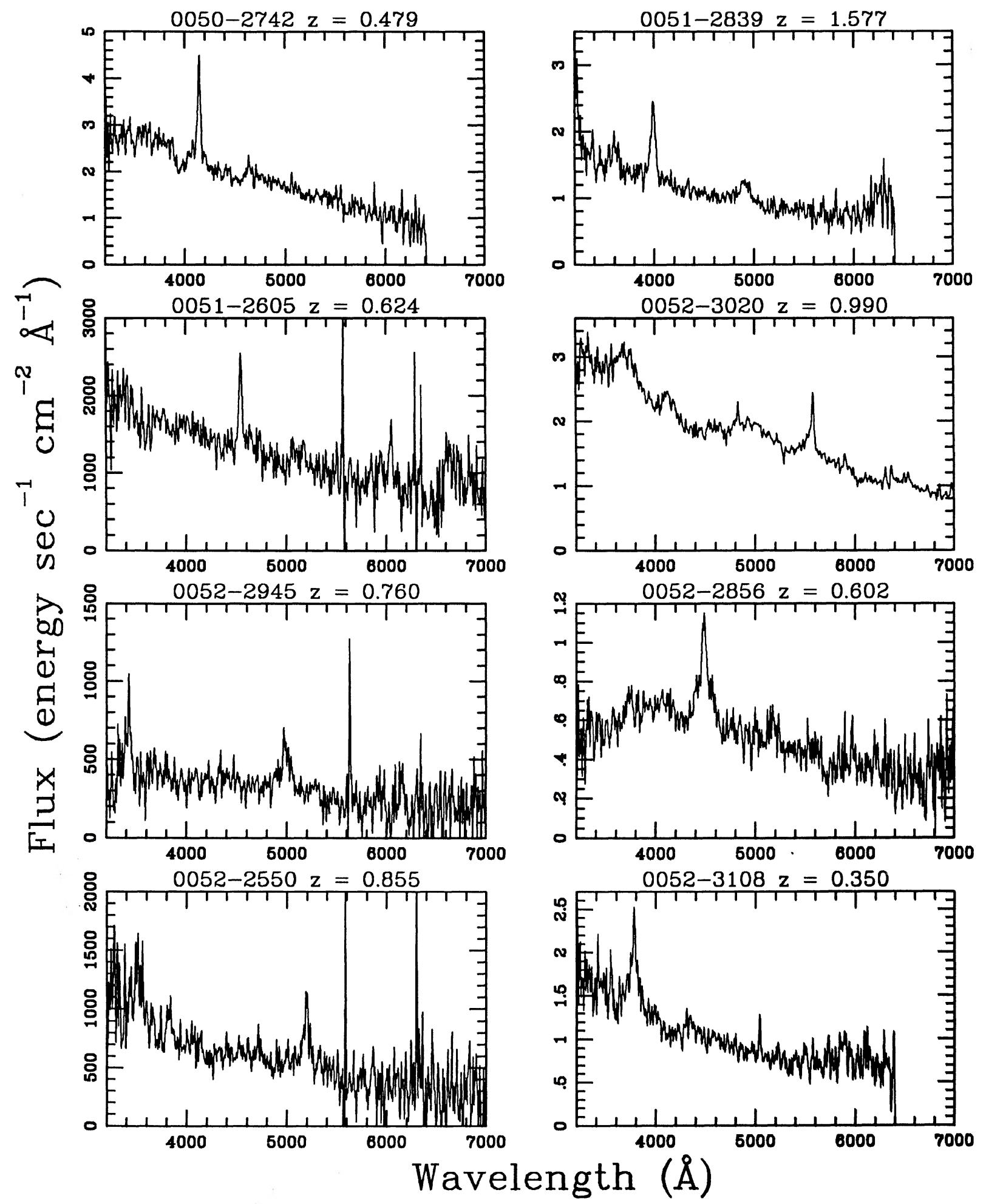

FIG. 1. (continued) 


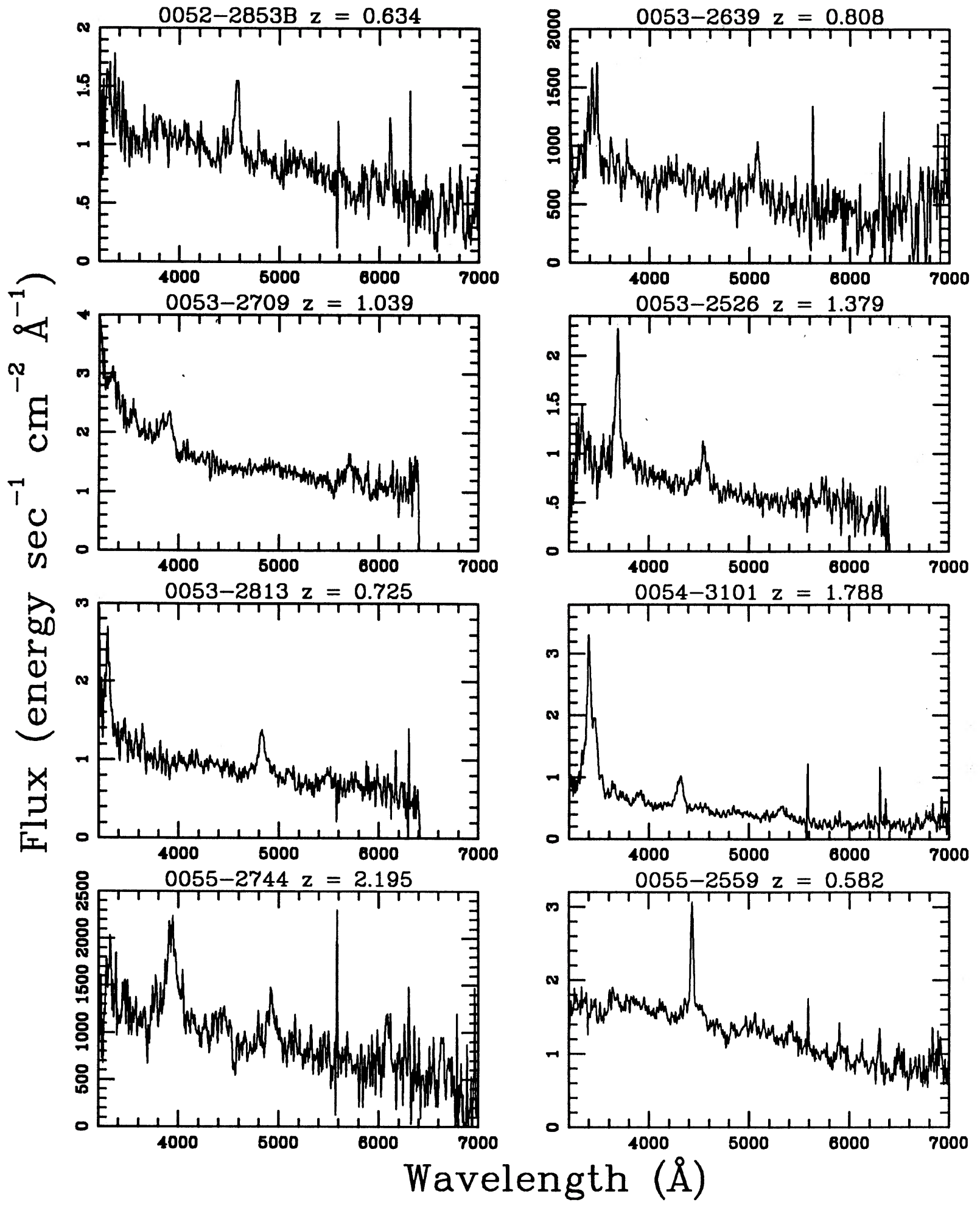

FIG. 1. (continued) 

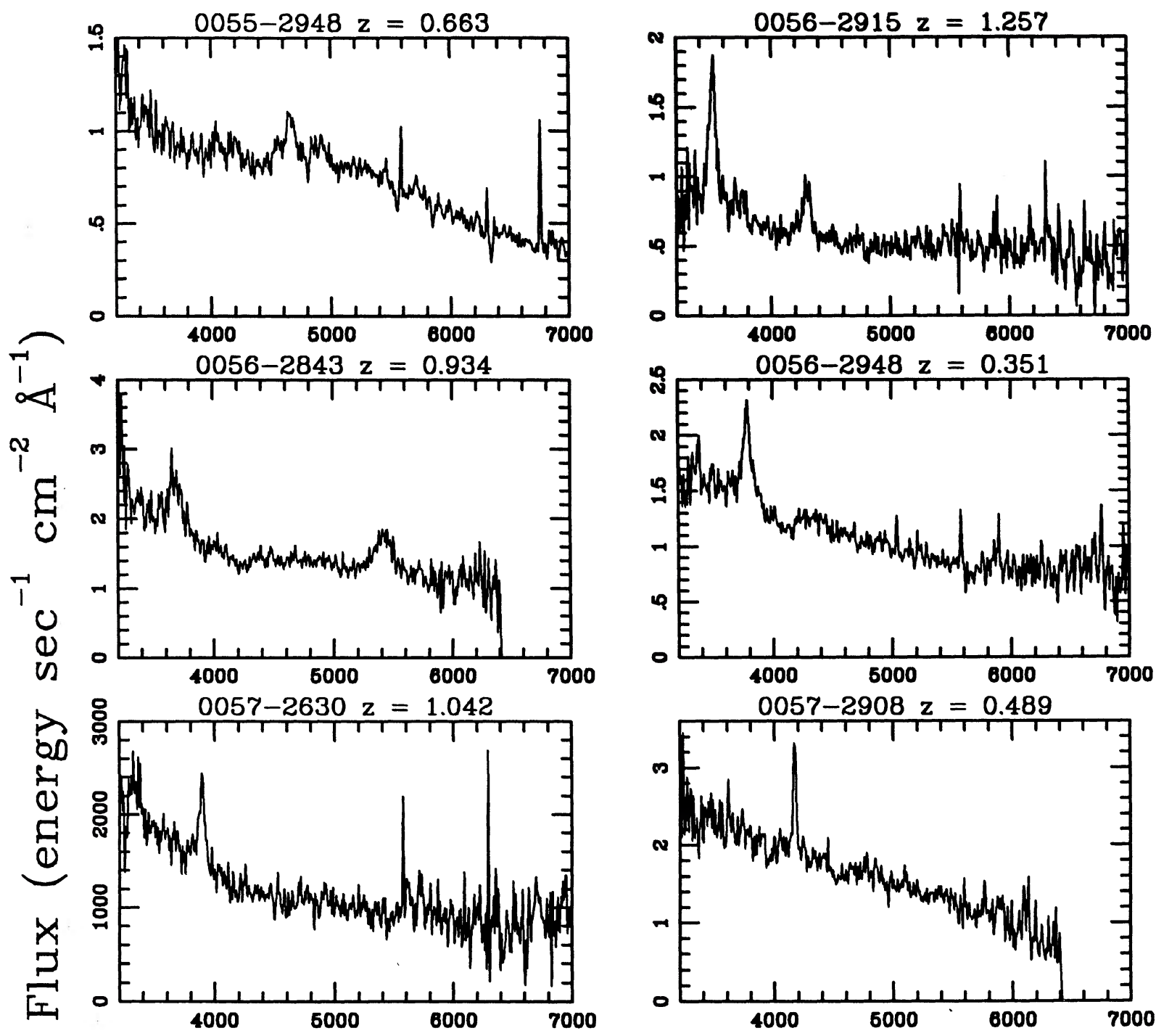

$0057-2729 \mathrm{z}=1.203$

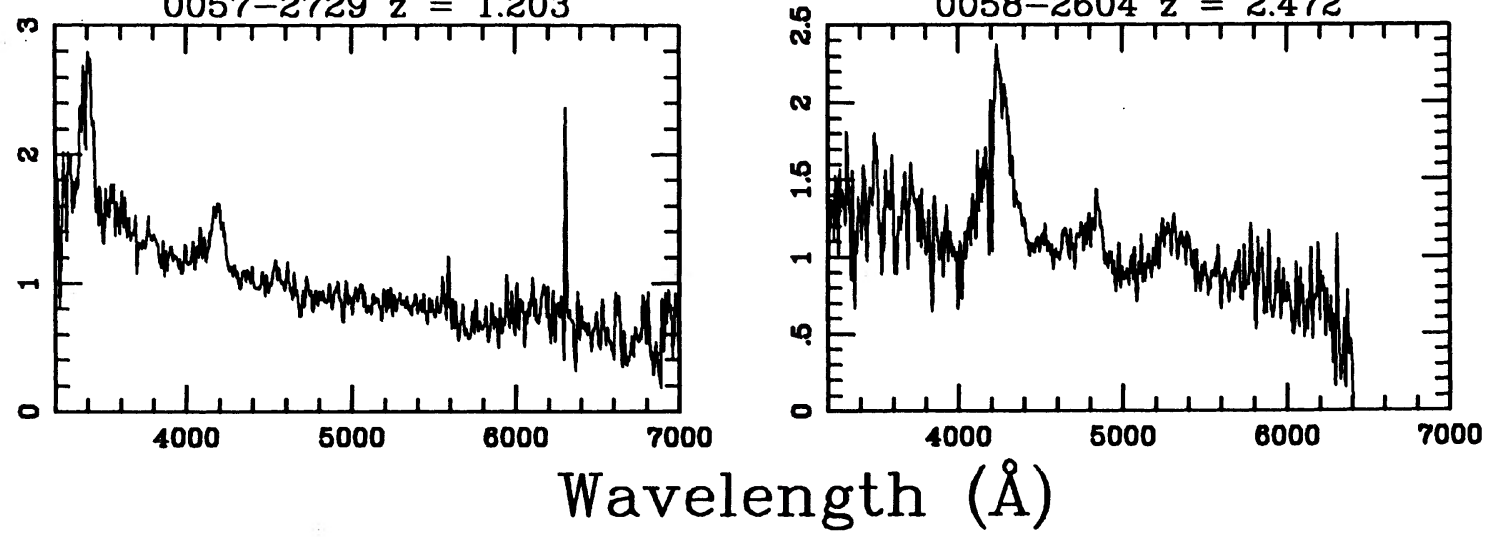

FIG. 1. (continued) 


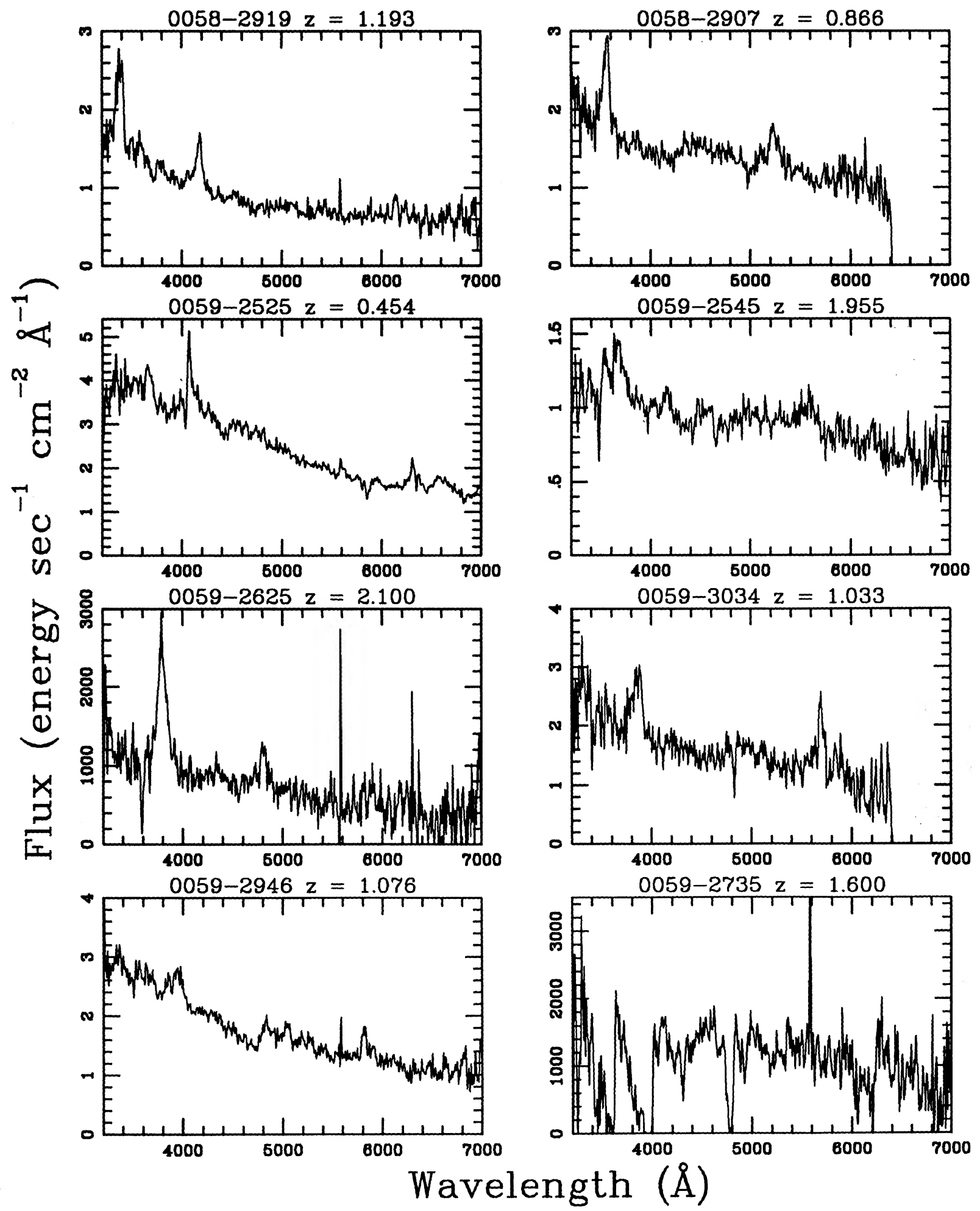

Fig. 1. (continued) 


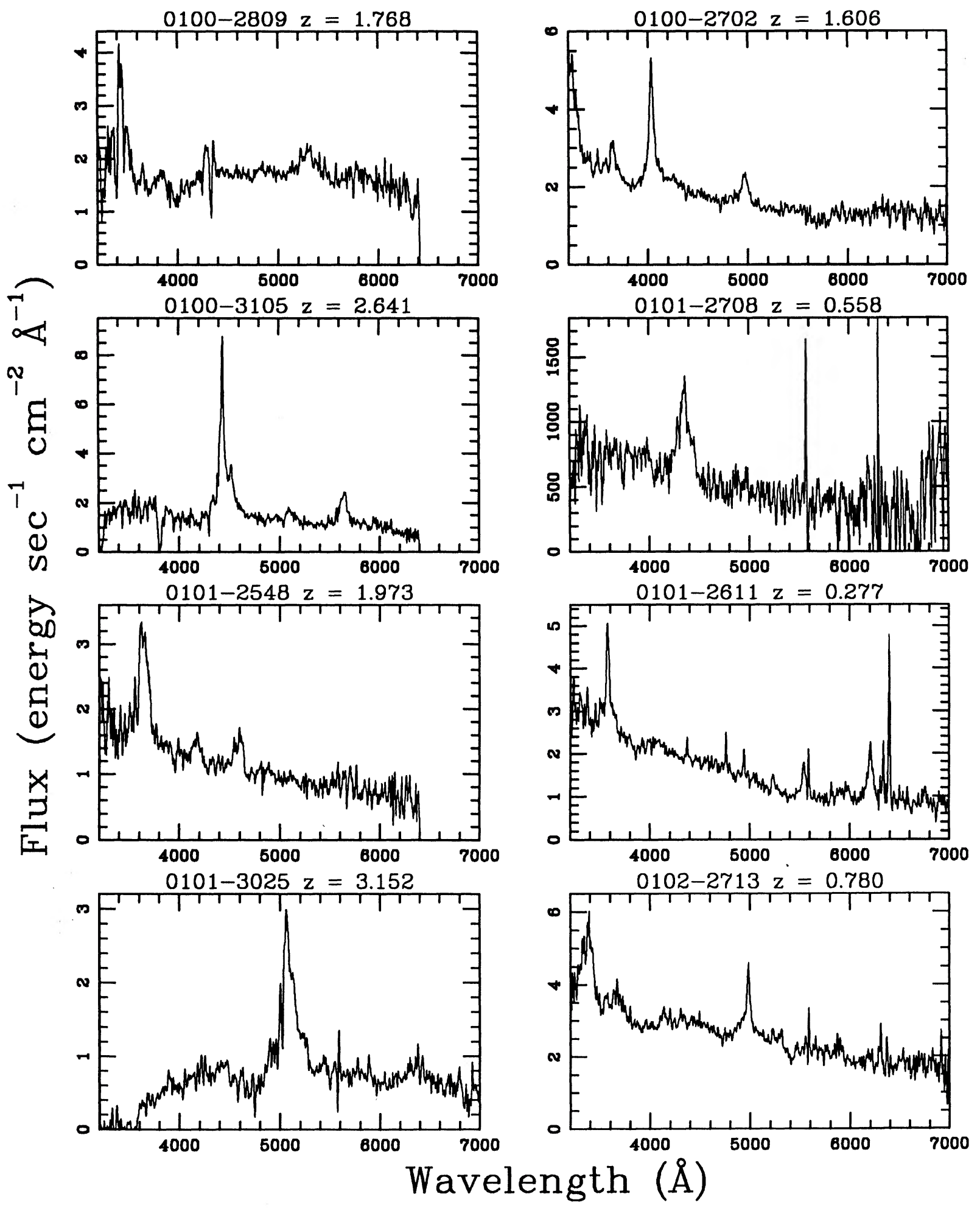

FIG. 1. (continued) 


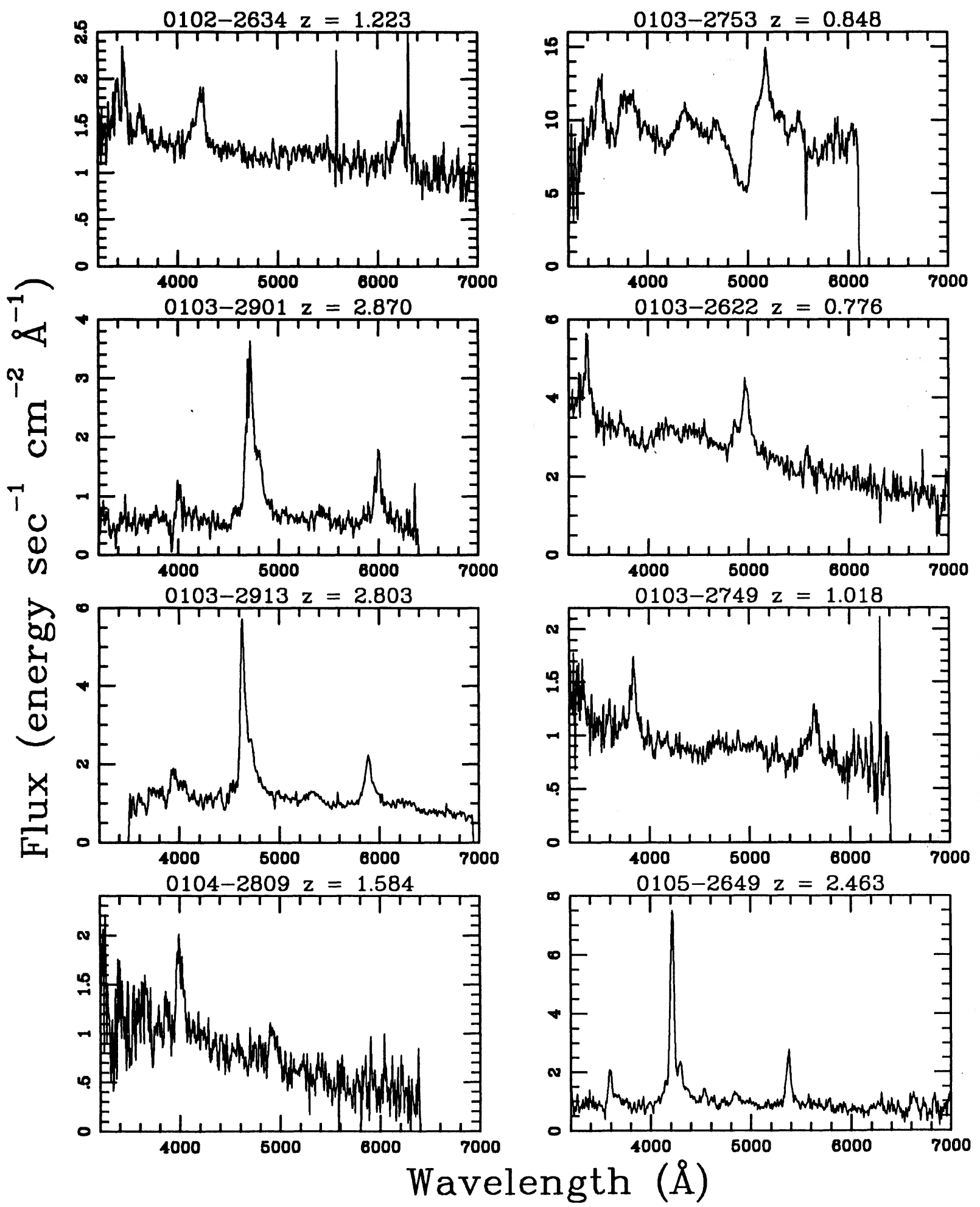

FIG. 1. (continued) 


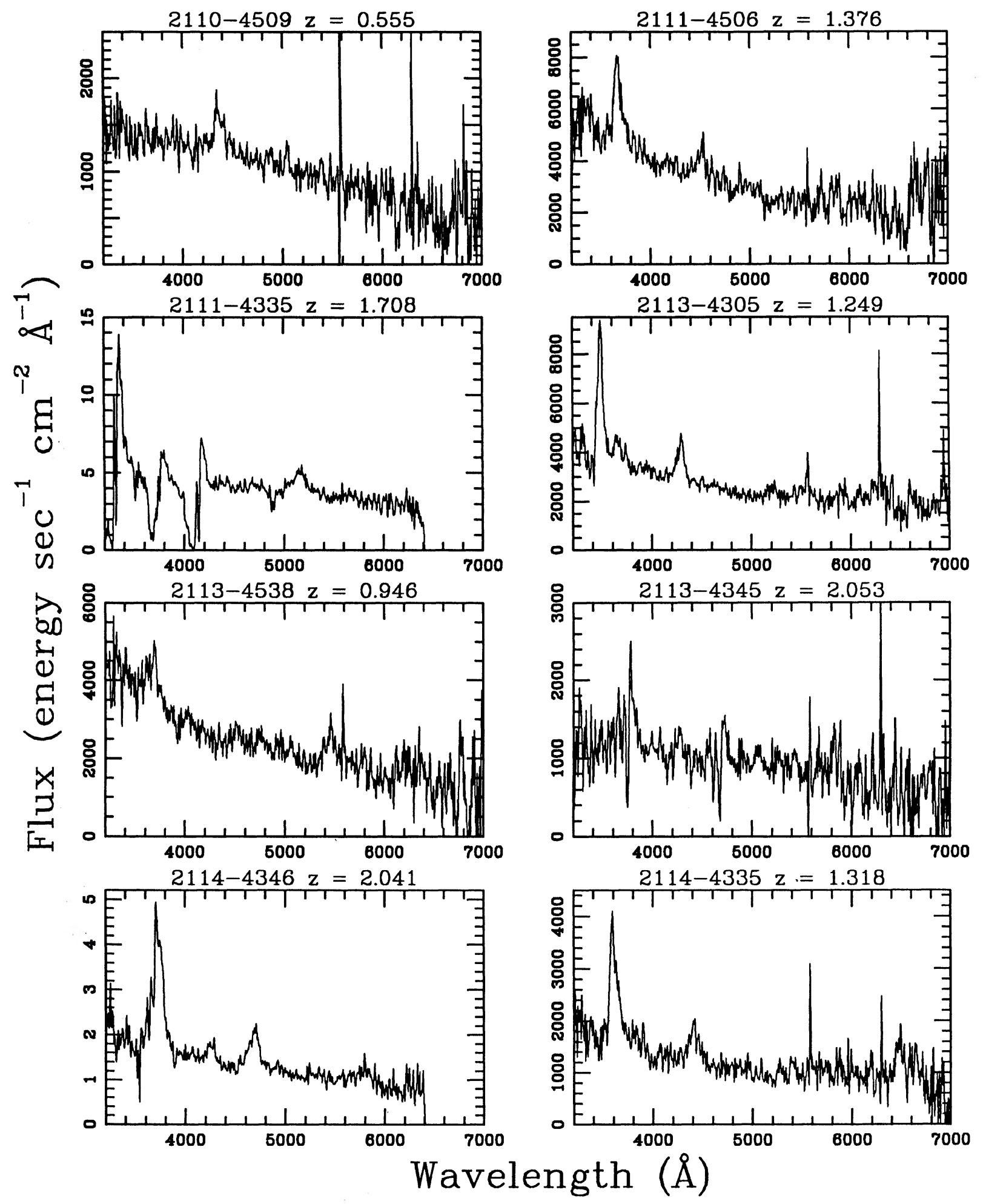

FIG. 1. (continued) 


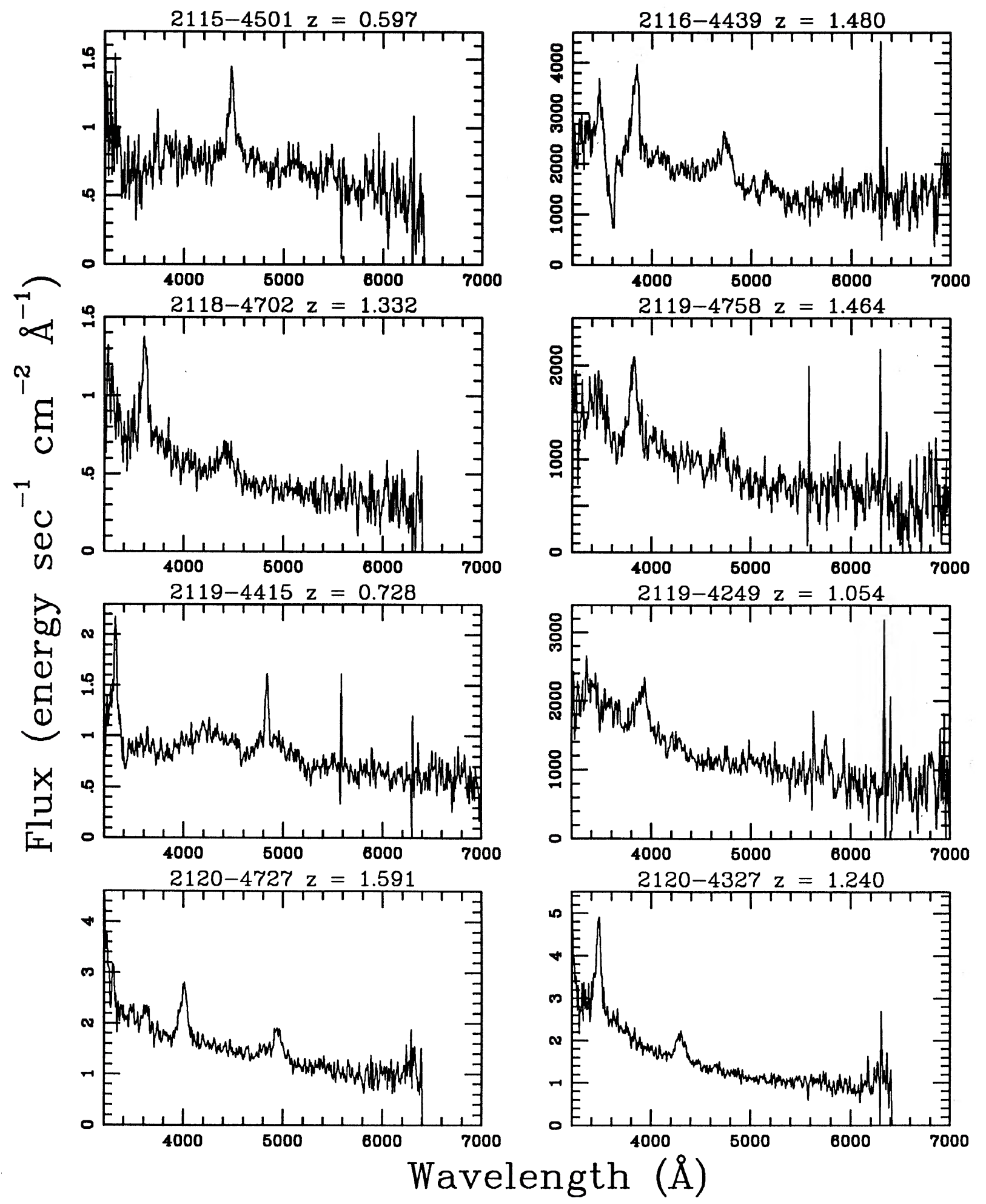

FIG. 1. (continued) 


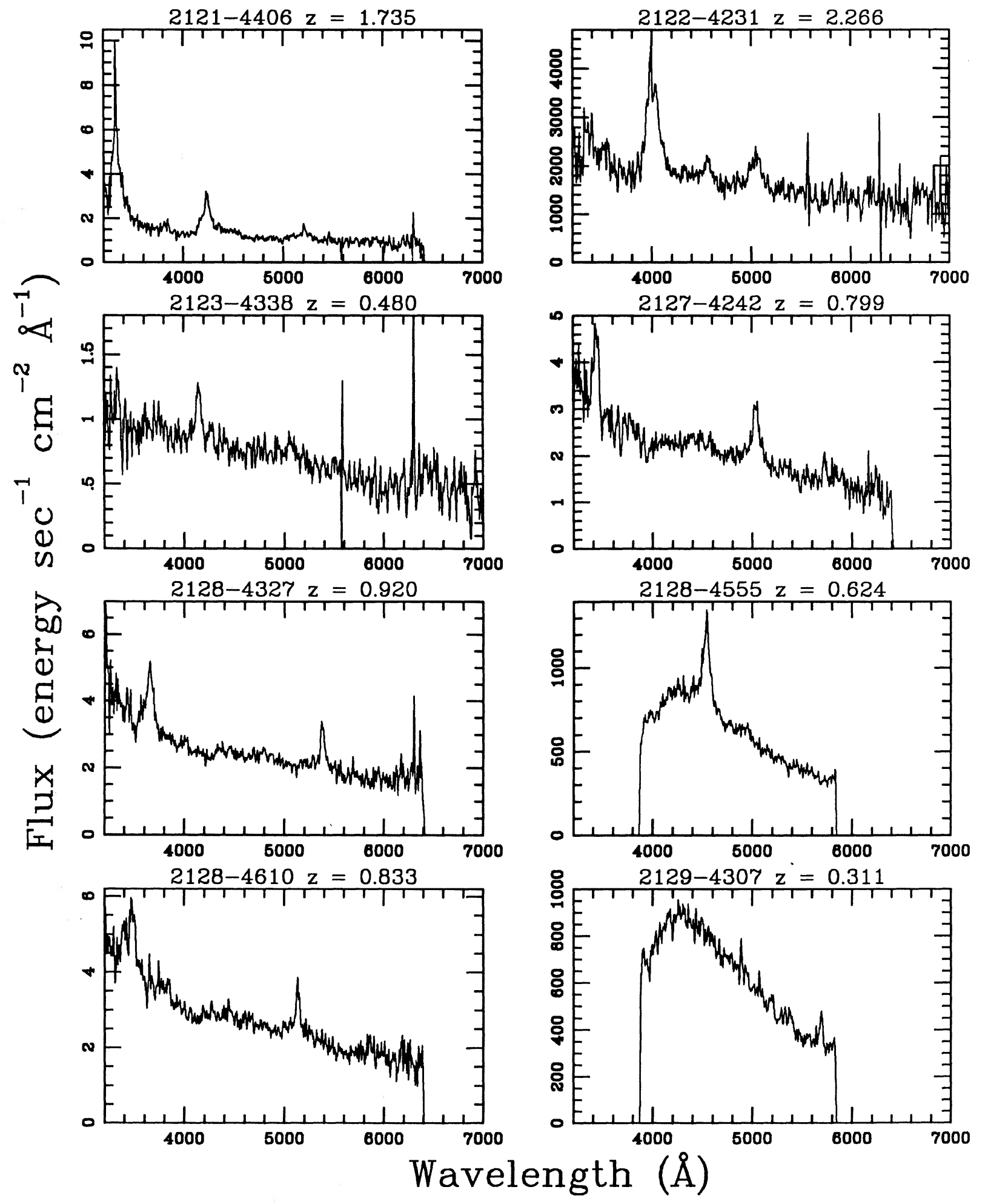

Fig. 1. (continued) 


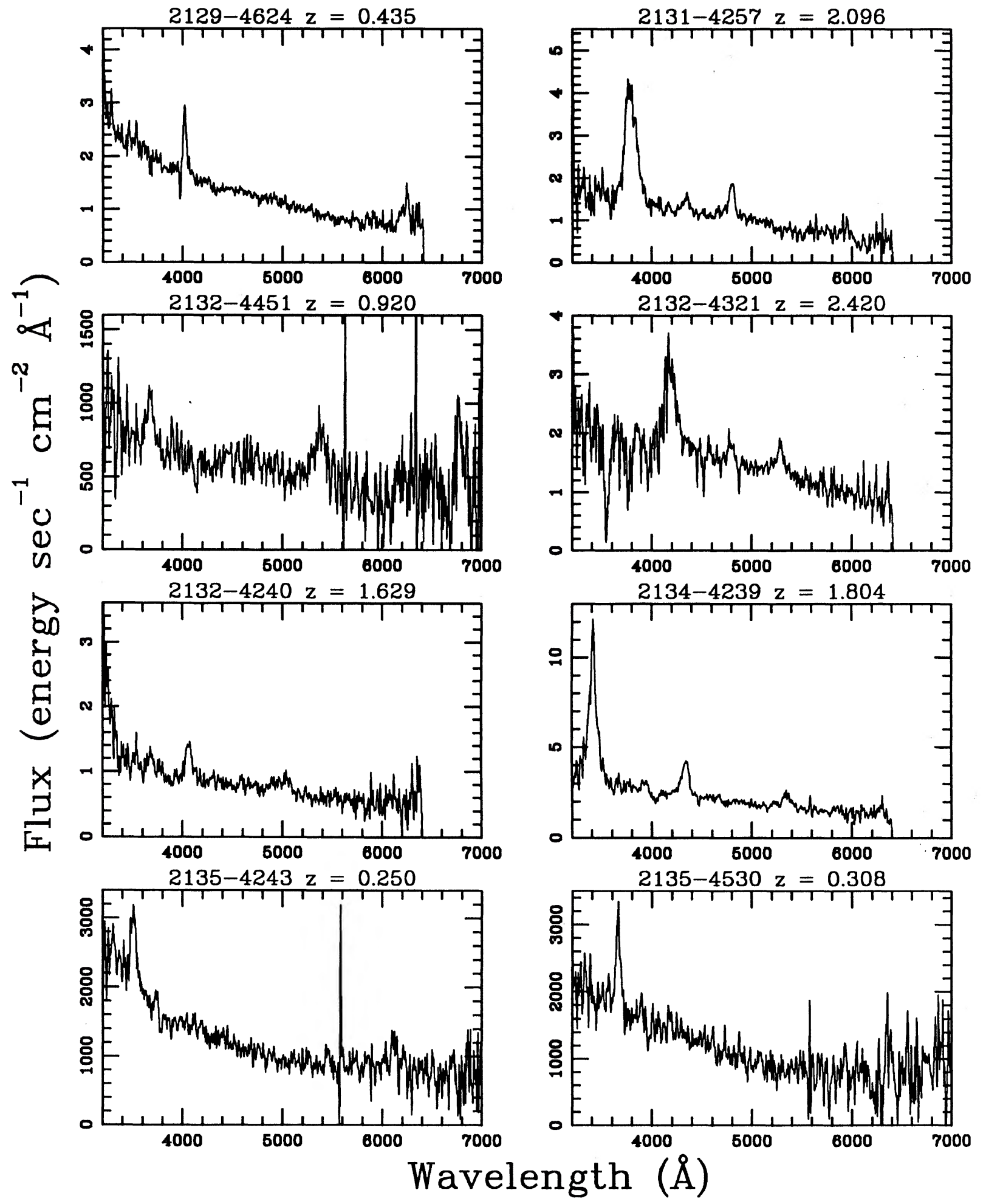

Fig. 1. (continued) 


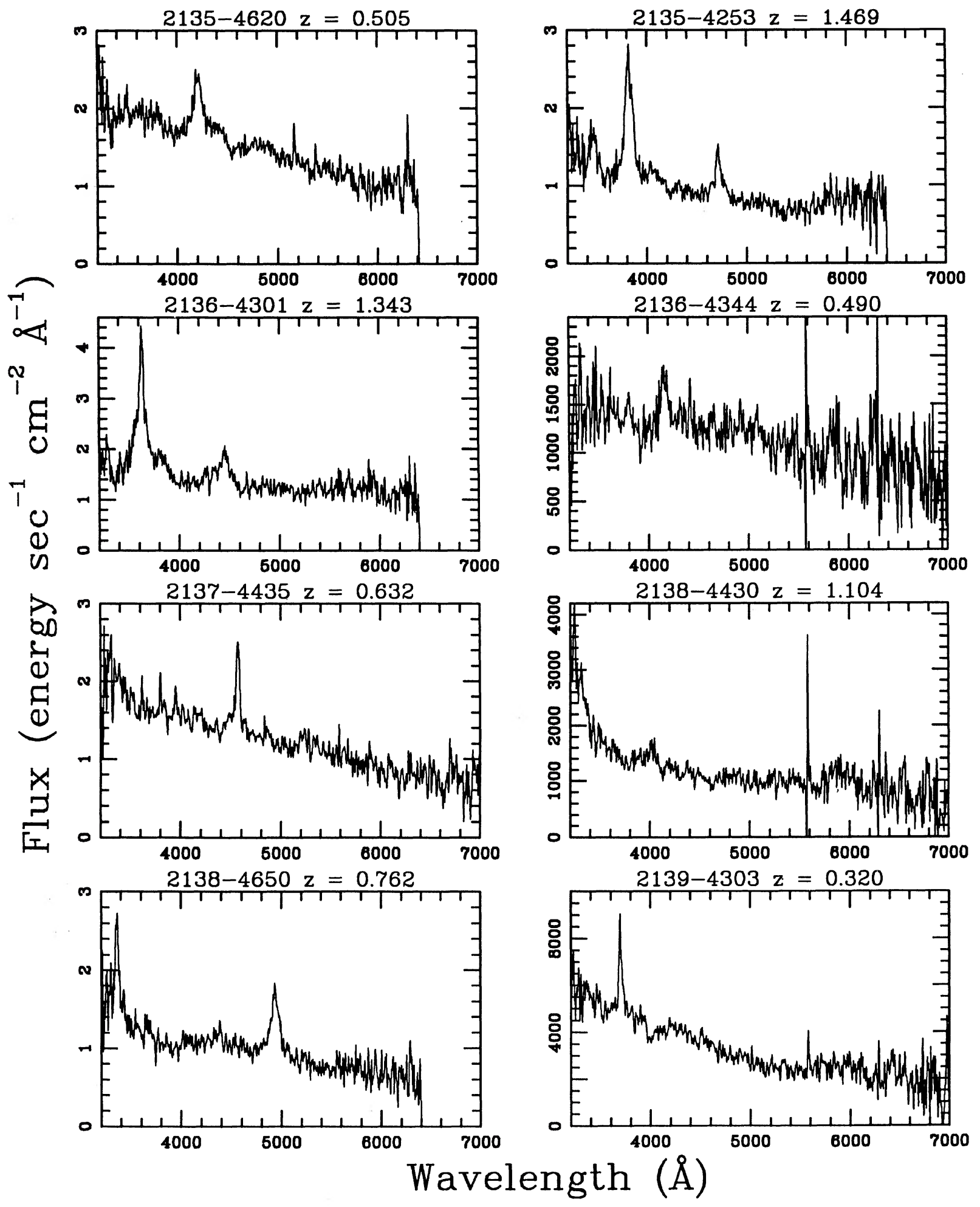

FIG. 1. (continued) 


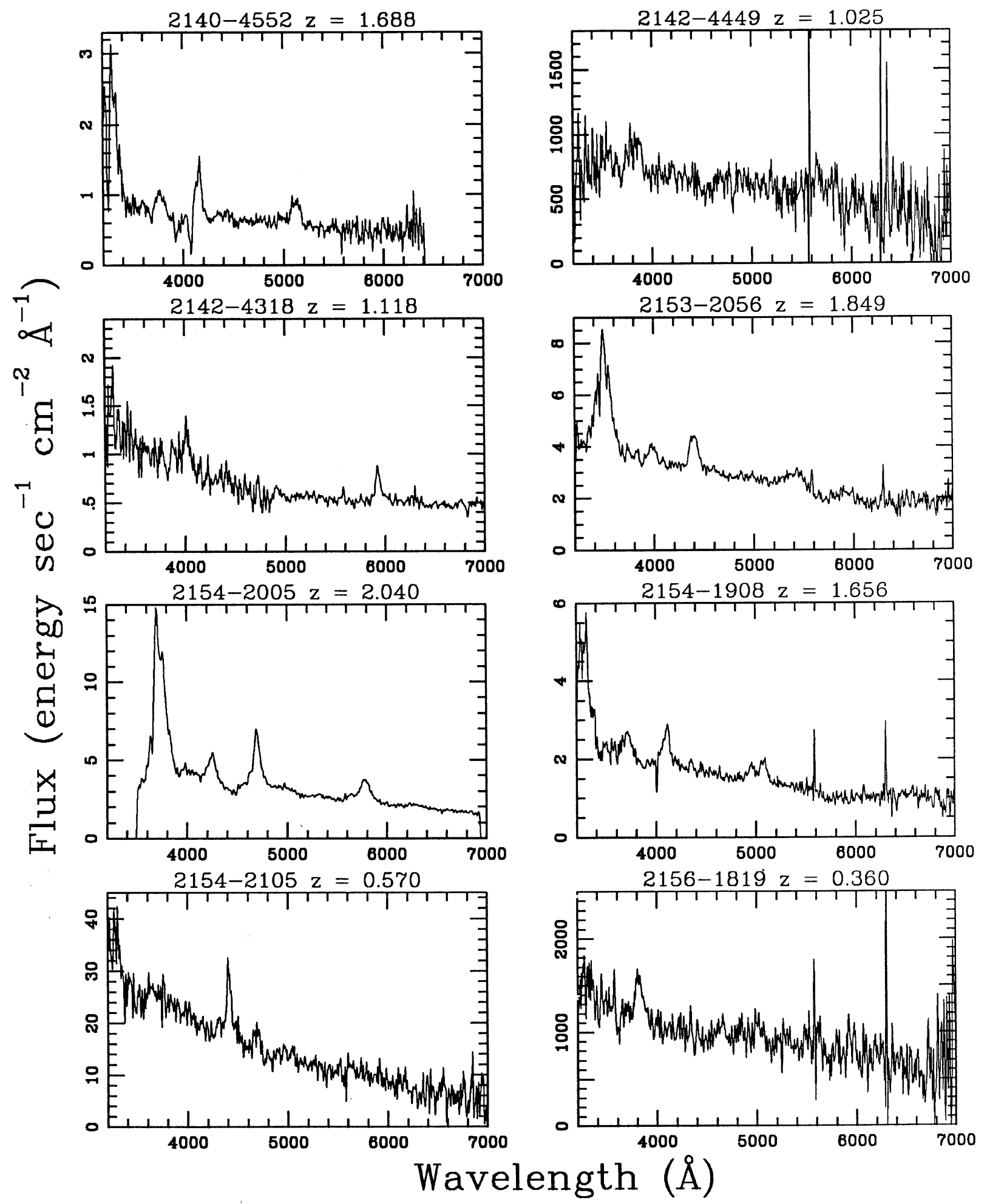

FIG. 1. (continued) 


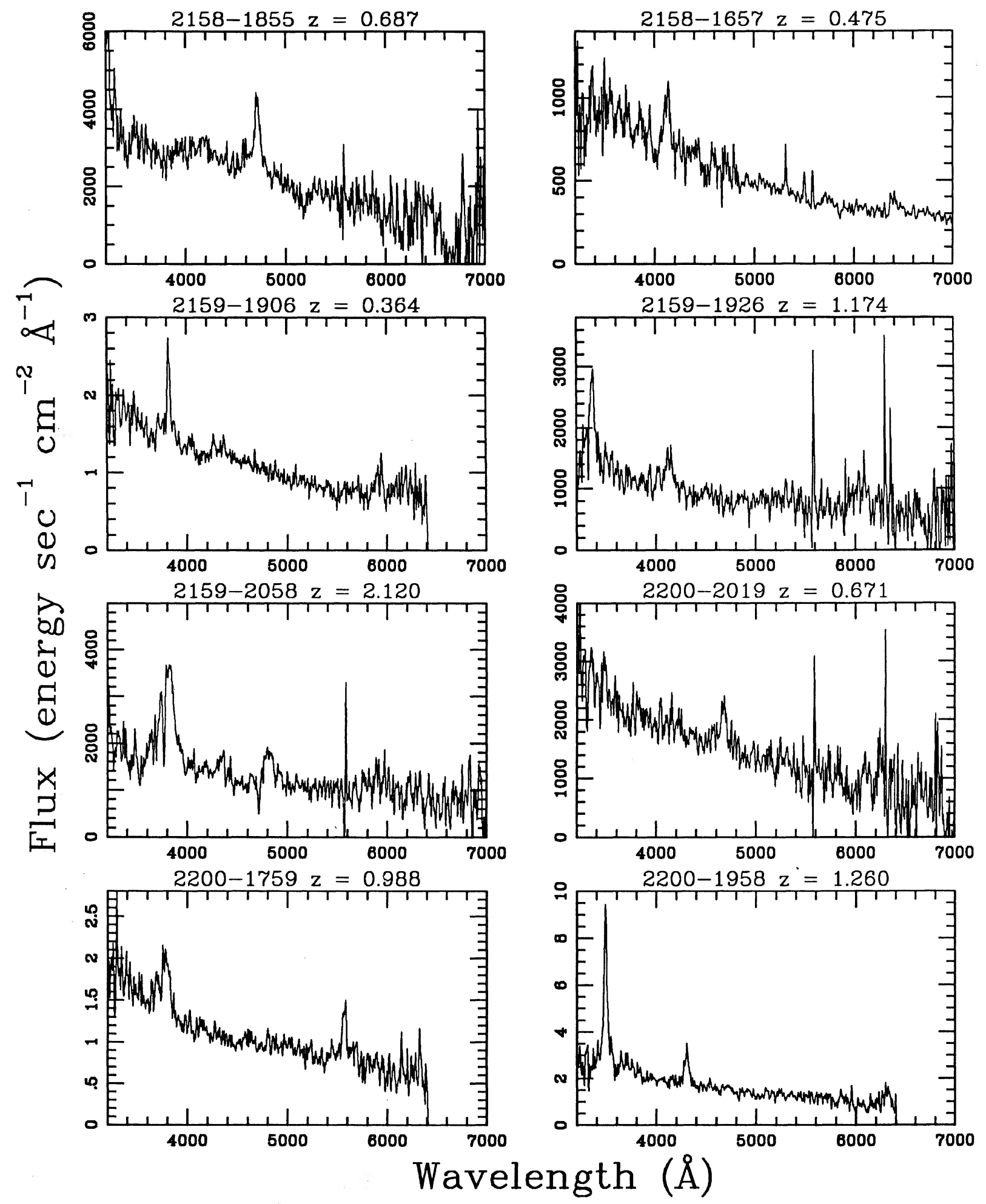

FIG. 1. (continued) 


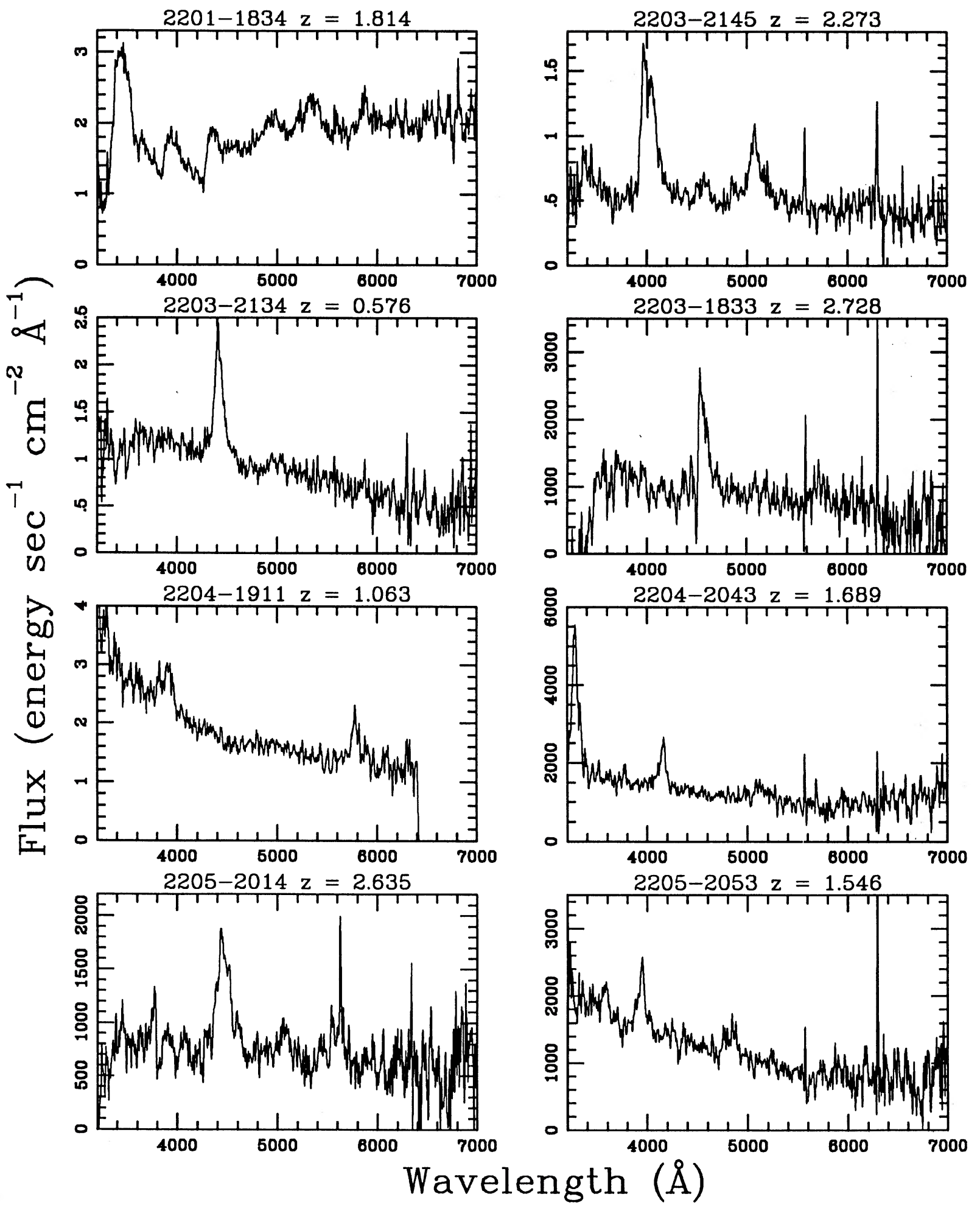

FIG. 1. (continued) 


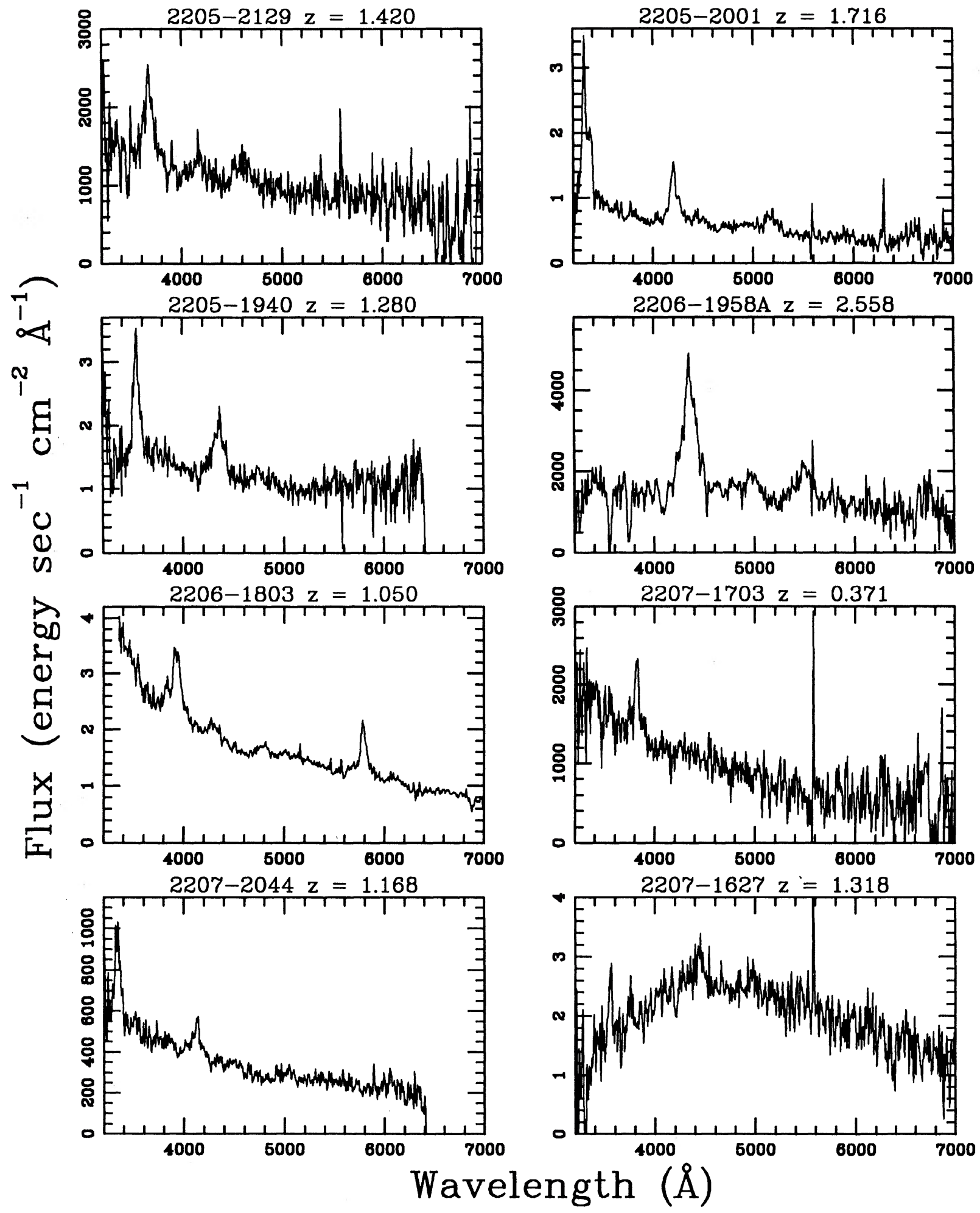

FIG. 1. (continued) 


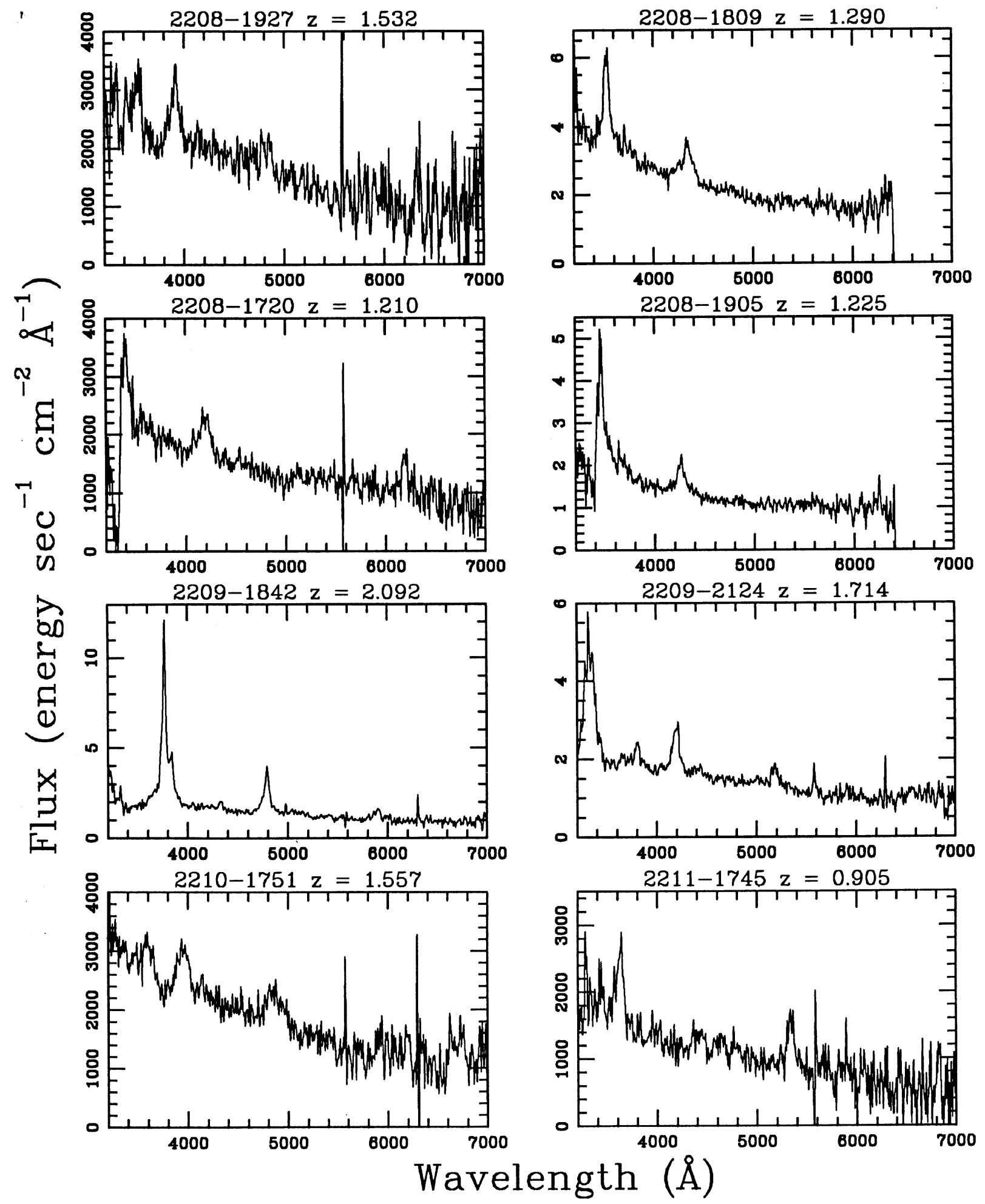

FIG. 1. (continued) 

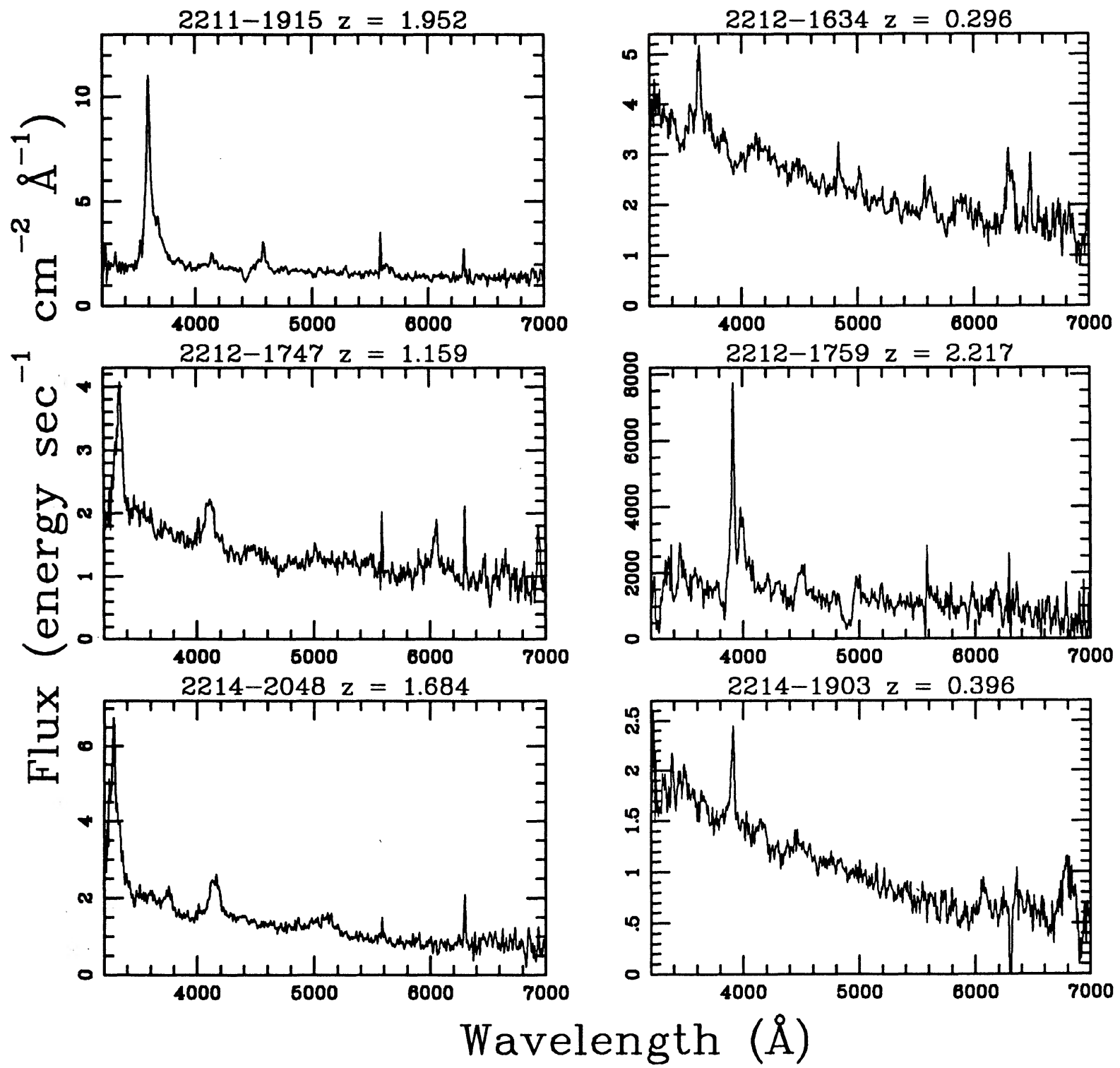

FIG. 1. (continued) 

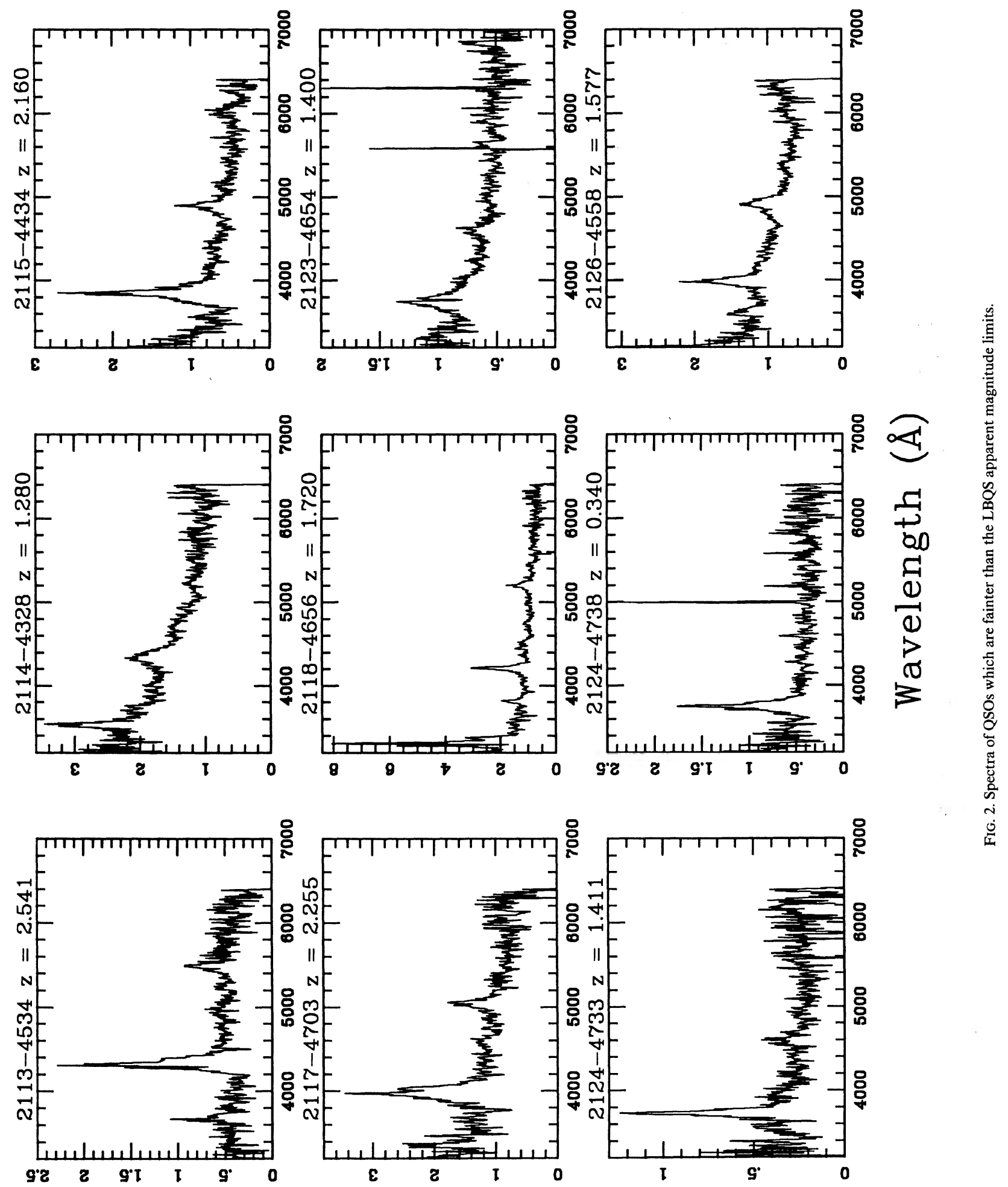

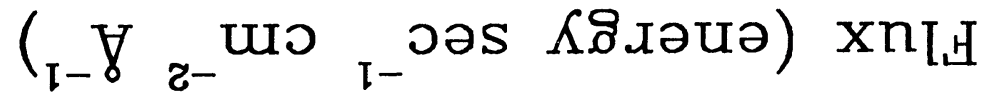



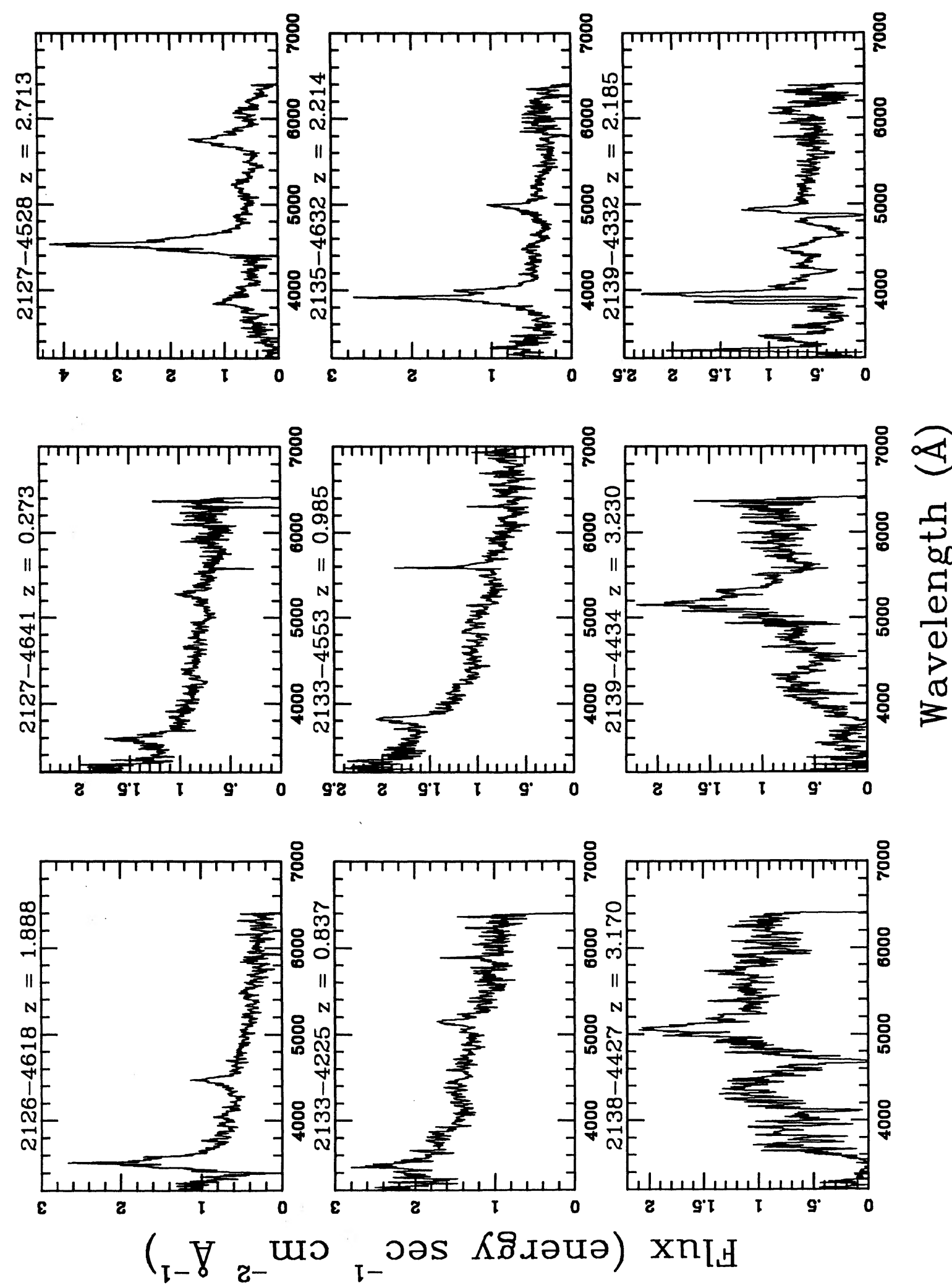
TABLE 3. QSOs Fainter than magnitude limit.

\begin{tabular}{lllllll}
\hline \hline Designation & R.A. (1950) & Dec. (1950) & $\mathrm{z}_{\text {em }}$ & B J & UT Date & Comment \\
\hline $2113-4534$ & 211344.0 & -453417 & 2.541 & 18.9 & $08 / 28 / 87$ & \\
$2114-4328$ & 211445.8 & -432814 & 1.280 & 18.7 & $08 / 30 / 87$ & \\
$2115-4434$ & 211518.1 & -443454 & 2.160 & 18.9 & $08 / 27 / 87$ & \\
$2117-4703$ & 211721.8 & -470347 & 2.255 & 19.0 & $08 / 30 / 87$ & \\
$2118-4656$ & 211811.9 & -465602 & 1.720 & 18.9 & $08 / 27 / 87$ & \\
$2123-4654$ & 212303.7 & -465452 & 1.400 & 18.7 & $09 / 01 / 89$ & \\
$2124-4733$ & 212417.9 & -473303 & 1.411 & 18.9 & $08 / 27 / 87$ & \\
$2124-4738$ & 212428.9 & -473839 & 0.340 & 18.7 & $08 / 29 / 87$ & \\
$2126-4558$ & 212633.7 & -455845 & 1.577 & 18.9 & $08 / 28 / 87$ & \\
$2126-4618$ & 212652.9 & -461859 & 1.888 & 18.8 & $08 / 22 / 87$ & \\
$2127-4641$ & 212707.2 & -464124 & 0.273 & 18.8 & $08 / 26 / 87$ & \\
$2127-4528$ & 212737.2 & -452857 & 2.713 & 18.8 & $08 / 22 / 87$ & \\
$2133-4225$ & 213328.6 & -422518 & 0.837 & 18.6 & $08 / 30 / 87$ & \\
$2133-4553$ & 213341.2 & -455300 & 0.985 & 18.9 & $09 / 01 / 89$ & \\
$2135-4632$ & 213506.5 & -463227 & 2.214 & 18.8 & $08 / 28 / 87$ & \\
$2138-4427$ & 213848.4 & -442708 & 3.170 & 18.9 & $08 / 26 / 87$ & d,L \\
$2139-4434$ & 213914.7 & -443401 & 3.230 & 18.7 & $08 / 27 / 87$ & L? \\
$2139-4332$ & 213922.3 & -433205 & 2.185 & 18.6 & $08 / 29 / 87$ & b \\
& & & & & & \\
\hline \hline
\end{tabular}
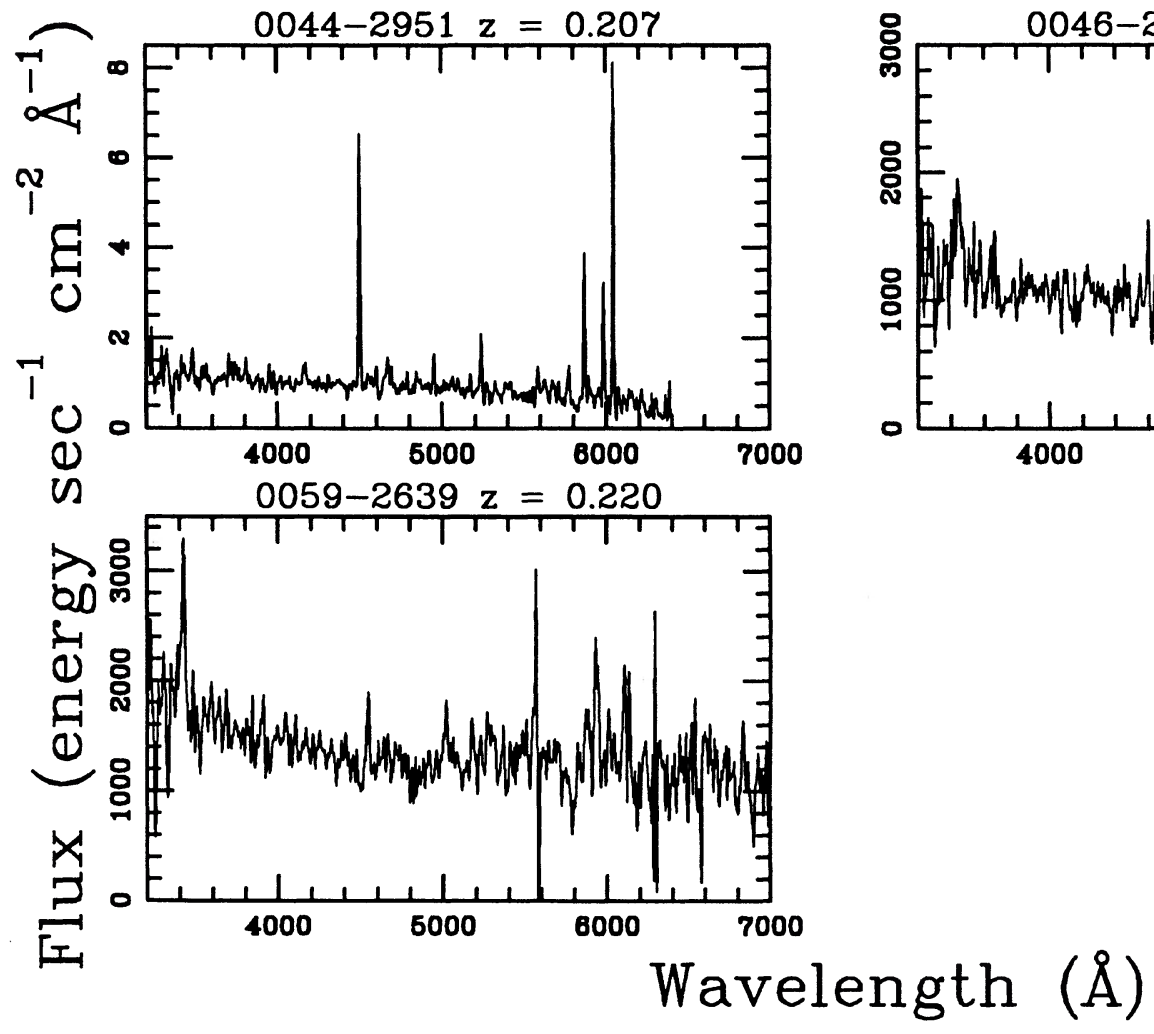

FIG. 3. Spectra of extragalactic objects with $z \geqslant 0.2$ and $M_{B_{J}}>-21.5$.
TABLE 4. AGNs with $z_{\mathrm{em}} \geqslant 0.2$ and $M_{J}$ absolute magnitude cutoff.

\begin{tabular}{lccccc}
\hline \hline Designation & R.A. (1950) & Dec. (1950) & $\mathrm{z}_{\mathrm{em}}$ & $\mathrm{B}_{\mathrm{J}}$ & UT Date \\
\hline $0044-2951$ & 004422.6 & -295121 & 0.207 & 18.3 & $08 / 28 / 87$ \\
$0046-2611$ & 004609.9 & -261139 & 0.234 & 18.5 & $09 / 09 / 88$ \\
$0059-2639$ & 005948.7 & -263955 & 0.220 & 18.2 & $09 / 09 / 88$ \\
\hline
\end{tabular}

TABLE 5. AGNs and emission-line galaxies.

\begin{tabular}{lccccc}
\hline \hline Designation & R.A. (1950) & Dec. (1950) & $\mathrm{z}_{\mathrm{em}}$ & B $_{\mathbf{J}}$ & UT Date \\
\hline $0052-2956$ & 005222.2 & -295621 & 0.199 & 18.8 & $09 / 09 / 88$ \\
$2131-4350$ & 213149.3 & -435027 & 0.030 & 17.2 & $09 / 09 / 88$ \\
$2140-4542$ & 214010.0 & -454229 & 0.171 & 16.7 & $09 / 01 / 89$ \\
$2150-1927$ & 215032.3 & -192749 & 0.137 & 17.5 & $10 / 13 / 88$ \\
$2154-1942$ & 215440.5 & -194202 & 0.154 & 18.5 & $09 / 10 / 88$ \\
$2154-1849$ & 215452.1 & -184917 & 0.139 & 16.6 & $08 / 26 / 87$ \\
$2157-1753$ & 2157.45 .7 & -175315 & 0.140 & 18.4 & $08 / 25 / 87$ \\
$2202-1724$ & 220244.2 & -172434 & 0.141 & 17.9 & $08 / 25 / 87$ \\
& & & & &
\end{tabular}

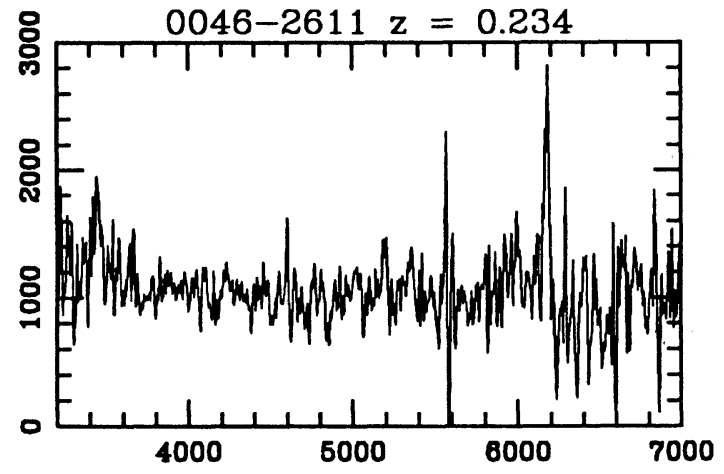


TABLE 6. Previously discovered QSOs not in LBQS.

\begin{tabular}{|c|c|c|c|c|c|c|c|c|c|c|c|}
\hline Designation & R.A. (1950) & Dec. $(1950)$ & $\mathrm{m}_{\text {pub }}$ & $\mathbf{B}_{\mathbf{J}}$ & Notes & Designation & R.A. (1950) & Dec. $(1950)$ & $\mathrm{m}_{\text {pub }}$ & $\mathrm{B}_{\mathbf{J}}$ & Notes \\
\hline $0043-267$ & 004338.4 & -264430 & 18.9 & 19.3 & $\mathbf{a}$ & $0100-261$ & 010033.1 & -261033 & 18.7 & 18.9 & $\mathbf{a}$ \\
\hline $0044-281$ & 004420.7 & -280753 & not given & 19.1 & $\mathbf{a}$ & $0100-289$ & 010050.8 & -285728 & 18.9 & 19.2 & $\mathbf{a}$ \\
\hline $0045-258$ & 004540.8 & -255131 & not given & 19.6 & $\mathbf{a}$ & $0101-268$ & 010115.8 & -265328 & 19.0 & 19.4 & $\mathbf{a}$ \\
\hline $0045-288$ & 004541.1 & -285030 & 18.9 & 18.9 & $\mathbf{a}$ & $0103-271$ & 010307.8 & -270737 & 19.0 & 18.9 & $\mathbf{a}$ \\
\hline $0045-260$ & 004549.9 & -260409 & 18.6 & 18.9 & $\mathbf{a}$ & $0104-275$ & 01042.1 & -273414 & 18.6 & 18.8 & $\mathbf{a}$ \\
\hline 0047-293 & 004706.2 & -292253 & not given & 19.4 & a & $0104-271$ & 010422.0 & -270751 & not given & 20.5 & $a, b$ \\
\hline 0048-298 & 004816.2 & -295236 & 19.0 & 19.2 & a & 0104-265 & 010422.8 & -263503 & not given & 19.8 & $a$ \\
\hline $0048-261$ & 004842.5 & -260836 & 18.2 & 19.6 & a & $2118-430$ & 211855.7 & -430338 & 18.43 & 18.7 & $\mathbf{a}$ \\
\hline $0048-297$ & 004855.0 & -294446 & 18.9 & 19.6 & a & Q2121-4510 & 212137.0 & -451055 & 18.00 & 18.9 & $a, f$ \\
\hline 0049-296 & 004913.0 & -293933 & 17.6 & - & $c, e$ & $2131-461$ & 213102.6 & -460931 & 18.47 & 18.8 & $\mathbf{a}$ \\
\hline $0049-272$ & 004929.4 & -271402 & 18.4 & 19.0 & $\mathbf{a}$ & $2132-452$ & 213202.9 & -451610 & 18.32 & 19.0 & $\mathbf{a}$ \\
\hline $0049-290$ & 004929.8 & -290041 & 18.8 & 18.9 & $\mathbf{a}$ & Q2132-4245A & 213204.6 & -424542 & 18.82 & 19.2 & $a, f$ \\
\hline $0049-276$ & 004944.7 & -273613 & 18.7 & 18.9 & $\mathbf{a}$ & $2133-439$ & 213345.7 & -435845 & 18.11 & 18.9 & $a, c$ \\
\hline $0050-281$ & 005010.2 & -280710 & 18.9 & 19.0 & a & Q2134-4601 & 213450.8 & -460149 & 17.83 & - & $c, e, f$ \\
\hline $0050-300$ & 005028.6 & -300100 & not given & 19.7 & $\mathbf{a}$ & Q2135-4400 & 213541.3 & -440049 & 18.30 & 18.9 & $a, f$ \\
\hline 0050-294 & 005037.0 & -292913 & 18.92 & 18.8 & a & $2152-211$ & 215237.5 & -210727 & 18.9 & 18.6 & $\mathbf{a}$ \\
\hline 0051-275 & 005114.0 & -273342 & 18.8 & 18.9 & a & $2153-204$ & 215347.2 & -202650 & 17.01 & 17.4 & c \\
\hline 0051-265 & 005125.4 & -262943 & not given & 19.9 & $a, b$ & 2154-204 & 2154 & -2024 & not given & - & g \\
\hline $0051-274$ & 005138.2 & -272627 & 18.90 & 18.9 & $a$ & $2154-205$ & 215449.1 & -203207 & 18.4 & 20.0 & $\mathbf{a}$ \\
\hline $0051-266$ & 005140.1 & -263718 & not given & 18.9 & $a, b$ & $2155-174$ & 215539.9 & -172859 & 18.85 & 18.6 & $\mathbf{a}$ \\
\hline $0051-280$ & 005143.2 & -280122 & not given & 20.3 & $\mathbf{a}$ & $2156-204$ & 215648.8 & -202624 & 18.5 & 18.6 & a \\
\hline $0051-281$ & 005146.8 & -281152 & 18.97 & 18.9 & $\mathbf{a}$ & $2157-200$ & 215721.7 & -200014 & 18.5 & 18.8 & a \\
\hline $0051-302$ & 005148.7 & -301211 & 19.0 & - & $\mathbf{c}, \mathbf{e}$ & $2157-180$ & 215745.3 & -180523 & 18.6 & 18.7 & $\mathbf{a}$ \\
\hline $0051-274$ & 005150.8 & -272444 & not given & 19.5 & $\mathbf{a}$ & $2158-214$ & 215808.2 & -212822 & 18.15 & 17.9 & c \\
\hline $0053-286$ & 005330.9 & -283626 & 18.6 & 18.9 & a & $2158-179$ & 215820.6 & -175434 & 18.9 & 18.7 & $\mathbf{a}$ \\
\hline $0053-284$ & 005359.9 & $\begin{array}{l}-282446 \\
-2733\end{array}$ & 18.25 & 19.6 & a & $2159-175$ & 215950.1 & -173059 & 19.0 & 19.3 & a \\
\hline 0054-275 & 005406.4 & -273336 & 18.92 & 19.0 & a & $2201-202$ & 220133.3 & -201352 & 18.25 & 18.8 & $\mathrm{a}$ \\
\hline $0055-269$ & 005532.6 & -265927 & 17.1 & 18.9 & a & $2202-185$ & 220235.6 & -183201 & 18.81 & 18.8 & $a$ \\
\hline $0055-284$ & 005538.4 & -282823 & 18.87 & 18.8 & a & $2202-189$ & 220254.1 & -185936 & 18.82 & 19.0 & a \\
\hline $0057-259$ & 005723.4 & -255543 & 19.0 & 19.0 & a & $2203-187$ & 220301.6 & -184627 & 18.27 & 18.5 & $\mathbf{a}$ \\
\hline $0057-288$ & 005750.7 & -285133 & $19.0^{`}$ & - & $\mathbf{c}, \mathbf{e}$ & $2203-188$ & 220325.7 & -185018 & 18.50 & 18.7 & a \\
\hline $0058-285$ & 005841.3 & -283101 & 19.0 & 18.8 & $\mathbf{a}$ & $2206-199$ & 220615.5 & -195905 & 18.65 & 18.6 & $\mathbf{a}$ \\
\hline $0058-267$ & 005843.4 & -264329 & 19.0 & 19.3 & $a$ & $2207-204$ & 220749.4 & -202911 & 18.6 & 18.1 & h \\
\hline $0059-269$ & 005957.1 & -265754 & 18.9 & 18.9 & a & $2210-167$ & 221048.3 & -164337 & 18.5 & 18.5 & a \\
\hline $0100-291$. & 010015.9 & -291020 & 19.0 & 19.2 & $a$ & & & & & & \\
\hline
\end{tabular}

Notes to TABLE 6

a - Object fainter than LBQS field limit.

$\mathrm{b}-\mathrm{HB}$ coordinates in error by more than 5 arcseconds.

c - Spectrum overlapped on objective-prism plate.

$\mathrm{d}$ - Object brighter than LBQS field limit.

e - Direct image confused with nearby object. Magnitude unreliable.

f - Cristiani et al. (1990), quoted magnitude for maximum brightness.

$\mathrm{g}$ - No object found near published position.

$\mathrm{h}$ - This object is a star. 
magnitude limit for the field in which they appear, and six have spectra that overlap with those of nearby objects and were not processed. Of the remaining two, one, $2207-204$, which was originally only tentatively identified by Savage \& Bolton (1979) as a QSO from its objective prism spectrum, we have shown to be a star. The other, identified in HB as $2154-204$, is listed therein only by its nominal coordinates, and no finding chart is presented in the discovery paper of Savage et al. (1985), the only reference to this object of which we are aware. We find no image within 20 arcsec of the quoted position on our direct plate.

Thus, the $18 \mathrm{LBQS}$ fields included in this paper and $\mathrm{Pa}$ pers I-IV contain 160 previously known QSOs whose spectra were processable and which lay within the LBQS magnitude range. Of these only one bona fide QSO, 1237-009 (cf. Paper III) and the BL Lac object $1210+121$ (cf. Paper I) have escaped our detection.

Figure 4 shows the redshift histogram for 1031 LBQS QSOs as published in Papers I-V. There is a small dip in the $0.8-1.0$ redshift bin. We have analyzed the statistical significance of this feature in the following manner. An estimate was made independently by two of us of the number of QSOs in the $0.8-1.0$ bin based on the QSO distribution excluding that bin. We both obtained 105, 17 more than the observed number. One can then ask: given a hypothetical smooth distribution of 1048 objects $(1031+17)$, and hence an intrinsic probability of $0.1002(105 / 1048)$ for objects to fall in the $0.8-1.0 \mathrm{bin}$, what is the probability of getting $\leqslant 88$ objects in the $0.8-1.0$ bin from a sample of 1031 observed? The binomial probability one derives is $6 \%$.

The absence of discontinuities significant at the $\leqslant 5 \%$ level in this histogram at any redshift, and the extended range of redshifts covered is an independent qualitative indicator of the success of the LBQS selection procedures. Minor revisions of the photometric calibration and the extent of the scanned areas have occurred since the publication of Paper I. To ensure that the information necessary for statistical studies using the LBQS is readily accessible, we will produce a summary paper (Hewett et al., in preparation) giving details of all the QSOs making up the final LBQS sample.

Note added in proof. Recently, L. Campusano [AJ, 102, 502 (1991)] presented spectra for 35 QSOs in the SGP. Of

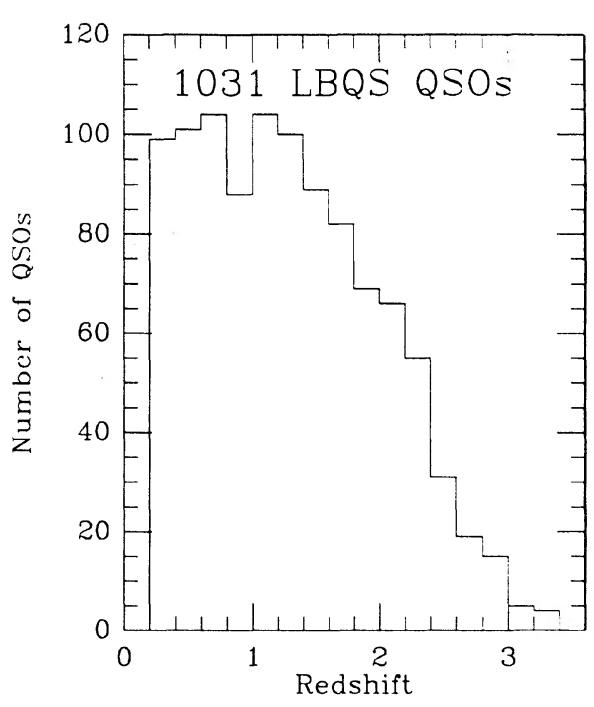

FIG. 4. Redshift histogram of the LBQS, including all objects in this paper and those in Papers I-IV.

those satisfying the LBQS magnitude and redshift criteria, only two $(01027-3012$ and $00505-2641)$ are not listed herein. Both are in unprocessable areas of the plates.

We thank Fernando Lopez and Angel Guerrero for their expert assistance at the duPont telescope. The LBQS is supported by National Science Foundation Grant Nos. AST90-01181 and 90-05117, for which we are grateful. The LBQS would not have been possible without the active support of the United Kingdom Schmidt Telescope Unit and the staff of the Automated Plate Measuring facility. We further acknowledge the computing support provided by the Starlink Project which is funded by the UK SERC. F. H. C. gratefully acknowledges the hospitality of the Institute of Astronomy and the support of a Royal Society Guest Research Fellowship. P. J. F. was supported by an SERC postgraduate studentship.

\section{REFERENCES}

Boyle, B. J., Fong, R., Shanks, T., and Peterson, B. A. 1990, MNRAS, 243, 1

Chaffee, F. H., Foltz, C. B., Hewett, P. C., Francis, P. F., Weymann, R. J., Morris, S. L., Anderson, S. F., and MacAlpine, G. M. 1991, AJ, 102, 461 (Paper IV)

Cristiani, S., Hawkins, M., Iovino, A., Pierce, M., and Shaver, P. 1990, MNRAS, 245, 493

Foltz, C. B., Chaffee, F. H., Hewett, P. C., MacAlpine, G. M., Turnshek, D. A. Weymann, R. J., and Anderson, S. F. 1987, AJ, 94, 1423 (Paper I)

Foltz, C. B., Chaffee, F. H., Hewett, P. C., Weymann, R. J., Anderson, S. F., and MacAlpine, G. M. 1989, AJ, 98, 1959 (Paper II)

Francis, P. J., Hewett, P. C., Foltz, C. B., Chaffee, F. H., Weymann, R. J., and Morris, S. L. 1991, ApJ, 373, 465

Hewett, P. C., Foltz, C. B., Chaffee, F. H., Francis, P. J., Weymann, R. J., Morris, S. L., Anderson, S. F., and MacAlpine, G. M. 1991, AJ, 101, 1121 (Paper III)

Hewitt, A., and Burbidge, G. 1987, ApJS, 63, 1

Hewitt, A., and Burbidge, G. 1989, ApJS, 69, 1

Savage, A., and Bolton, J. G. 1979, MNRAS, 188, 599

Savage, A., Clowes, R. G., Cannon, R. D., Cheung, K., Smith, M. G., Boksenberg, A., and Wall, J. V. 1985, MNRAS, 213, 485

United Kingdom Schmidt Telescope Handbook 1983, Royal Observatory Edinburgh 